\title{
QUANTITATIVE FLATNESS RESULTS AND $B V$-ESTIMATES FOR STABLE NONLOCAL MINIMAL SURFACES
}

\author{
ELEONORA CINTI, JOAQUIM SERRA, AND ENRICO VALDINOCI
}

\begin{abstract}
We establish quantitative properties of minimizers and stable sets for nonlocal interaction functionals, including the $s$-fractional perimeter as a particular case.

On the one hand, we establish universal $B V$-estimates in every dimension $n \geqslant 2$ for stable sets. Namely, we prove that any stable set in $B_{1}$ has finite classical perimeter in $B_{1 / 2}$, with a universal bound. This nonlocal result is new even in the case of $s$-perimeters and its local counterpart (for classical stable minimal surfaces) was known only for simply connected two-dimensional surfaces immersed in $\mathbb{R}^{3}$.

On the other hand, we prove quantitative flatness estimates for minimizers and stable sets in low dimensions $n=2,3$. More precisely, we show that a stable set in $B_{R}$, with $R$ large, is very close in measure to being a half space in $B_{1}$-with a quantitative estimate on the measure of the symmetric difference. As a byproduct, we obtain new classification results for stable sets in the whole plane.
\end{abstract}

\section{Contents}

1. Introduction 2

1.1. The mathematical framework of nonlocal perimeter functionals

1.2. Motivations of nonlocal perimeters 7

1.3. Statement of the main results 11

2. Preliminary results 18

3. Proof of Theorems 1.7 and 1.9

4. Proof of Theorems 1.14 and 1.19

5. Energy estimates with perturbed kernels 41

6. Existence and compactness of minimizers 44

Appendix: Integral formulas for sets of finite perimeter

Acknowledgements

References

2010 Mathematics Subject Classification. 49Q05, 35R11, 53A10.

Key words and phrases. Nonlocal minimal surfaces, existence and regularity results. 


\section{INTRODUCTION}

In this paper we establish quantitative properties of minimizers and stable sets of nonlocal interaction functionals of perimeter type. We consider very general possibly anisotropic and not scaling invariant functionals - including, as particular cases, the fractional $s$-perimeter and its anisotropic version, introduced respectively in [11] and [30].

The results that we obtain can be grouped, roughly speaking, into the following categories:

- Local $B V$-estimates (universal bounds for the classical perimeter) and sharp energy estimates for minimizers and stable sets,

- Existence results and compactness of minimizers,

- Quantitative flatness results.

Before giving the most general statements of the results in the paper, we just state them for the case of fractional $s$-perimeter. Even in this very particular case, the results are new and interesting in themselves.

The precise setting of the (most general) nonlocal perimeter functionals that we consider will be discussed in Subsection 1.1. In particular, in the forthcoming Definitions 1.5 and 1.6 we precise the notions of minimizers and stable sets. Our results are stated in their full generality later on in Subsection 1.3 -after having given in Subsection 1.2 several concrete motivations for the problems under consideration.

We next state, in the case of the $s$-perimeter, our main $B V$-estimate. This result is a particular case of our Theorem 1.7. It gives a universal bound on the classical perimeter in $B_{1 / 2}$ of any stable minimal set in $B_{1}$. As said above, the precise notion of stable solution will be given in Definition 1.6, and it is an appropriate weak formulation of the nonnegativity of the second variation of the functional.

Theorem 1.1. Let $s \in(0,1), R>0$ and $E$ be a stable set in the ball $B_{2 R}$ for the nonlocal s-perimeter functional. Then, the classical perimeter of $E$ in $B_{R}$ is bounded by $C R^{n-1}$, where $C$ depends only on $n$ and $s$.

Moreover, the s-perimeter of $E$ in $B_{R}$ is bounded by $C R^{n-s}$.

Moreover, as a consequence of Theorem 1.7, we establish the same result for the anisotropic fractional perimeter considered in [30].

To better appreciate Theorem 1.1 let us compare it with the best known similar results for classical minimal surfaces. A universal perimeter estimate for (local) stable minimal surfaces is only known for the case of two-dimensional stable minimal surfaces that are simply connected and immersed in $\mathbb{R}^{3}$. Conversely, the perimeter estimate in our Theorem 1.1 holds in every dimension and without topological constraints. The perimeter estimate for the classical case is a result due to Pogorelov [35], and Colding and Minicozzi [18] - see also [32, Theorem 2] and [44, Lemma 34], it reads as follows 
Theorem 1.2 ([35, 18]). Let $D$ be a simply connected, immersed, stable minimal disk of geodesic radius $r_{0}$ on a minimal (two-dimensional) surface $\Sigma \subset \mathbb{R}^{3}$, then

$$
\pi r_{0}^{2} \leqslant \text { Area }(D) \leqslant \frac{4}{3} \pi r_{0}^{2}
$$

As said above, our estimate for nonlocal perimeters is stronger in the sense that we do not need $\partial E$ to be simply connected and immersed. In fact, an estimate exactly like ours can not hold for classical stable minimal surfaces since a large number of parallel planes is always a classical stable minimal surface with arbitrarily large perimeter in $B_{1}$.

The proof of Theorem 1.2 uses crucially the fact that for two-dimensional minimal surfaces the sum of the squares of the principal curvatures $\kappa_{1}^{2}+\kappa_{2}^{2}$ equals $2 \kappa_{1} \kappa_{2}=$ $-2 K$, where $K$ is the Gauß curvature - since on a minimal surface $\kappa_{1}+\kappa_{2}=0$. Then, the stability inequality reads as $\int_{D}|\nabla \xi|^{2}+2 K \xi^{2} \geqslant 0$. By plugging a suitable radial test function $\xi$ in this stability inequality, using the Gauß-Bonnet formula to relate $\int_{D_{r}} K$ and $\frac{d}{d r}$ Length $\left(\partial D_{r}\right)$, and integrating by parts in the radial variable, one proves the bound Area $(D) \leqslant \frac{4}{3} \pi r_{0}^{2}$. This elegant proof is unfortunately quite rigid and only applies to two-dimensional surfaces.

Having a universal bound for the classical perimeter of embedded minimal surfaces in every dimension $n \geqslant 4$ would be a decisive step towards proving the following well-known and long standing conjecture: The only stable embedded minimal (hyper)surfaces in $\mathbb{R}^{n}$ are hyperplanes as long as the dimension of the ambient space is less than or equal to \%. Indeed, it would open the door to use the monotonicity formula to prove that blow-downs of stable surfaces are stable minimal cones which are completely classified. On the other hand, without a universal perimeter bound, the sequence of blow-downs could have perimeters converging to $\infty$. In the same direction, we believe that our result in Theorem 1.1 can be used to reduce the classification of stable $s$-minimal surfaces in the whole $\mathbb{R}^{n}$ to the classification of stable cones

We note that our nonlocal estimate gives a control on the classical perimeter (i.e. the $B V$-norm of the characteristic function), which is stronger - both from the geometric and functional space perspective - than a control on the s-perimeter (i.e. on the $W^{s, 1}$ norm of the characteristic function). The sharp $s$-perimeter estimate stated in Theorem 1.1 is obtained as a consequence of the estimate for the classical perimeter using a standard interpolation.

Since it is well-known [5, 19, 14, 2] that the classical perimeter is the limit as $s \uparrow 1$ of the nonlocal $s$-perimeter (suitably renormalized), it is natural to ask whether our results give some informations in the limit case $s=1$. Unfortunately, our proof relies strongly on the nonlocal character of the $s$-perimeter and the constant $C$ appearing in Theorem 1.1 blows up as $s \uparrow 1$.

The more general forms of our $B V$-estimates have quite remarkable consequences regarding the existence and compactness of minimizers - see Theorem 1.13 and 
Lemma 6.7. These existence and compactness results are nontrivial since they apply in particular to some perimeter functionals that are finite on every measurable set. Thus, although all the perimeter functionals that we consider are lower semicontinuous, sequences of sets of finite perimeter are in principle not compact in $L^{1}$. Thanks to our $B V$-estimates, we can obtain robust compactness results that serve to prove existence of minimizers in a very general framework.

We next give our quantitative flatness estimate in dimension $n=2$ for the case of the $s$-perimeter. This result is a particular case of our Theorem 1.14 . It states that stable sets in a large ball $B_{R}$ are close to being a halfplane in $B_{1}$, with a quantitative control on the measure of the symmetric difference that decays to 0 as $R \rightarrow \infty$.

Theorem 1.3. Let the dimension of the ambient space be equal to 2 . Let $R \geqslant 2$ and $E$ be a stable set in the ball $B_{R}$ for the s-perimeter.

Then, there exists a halflplane $\mathfrak{h}$ such that $\left|(E \triangle \mathfrak{h}) \cap B_{1}\right| \leqslant C R^{-s / 2}$.

Moreover, after a rotation, we have that $E \cap B_{1}$ is the graph of a measurable function $g:(-1,1) \rightarrow(-1,1)$ with osc $g \leqslant C R^{-s / 2}$ outside a "bad" set $\mathcal{B} \subset(-1,1)$ with measure $C R^{-s / 2}$.

The previous result provides a quantitative version of the classification result in [37] which says that if $E$ is a minimizer of the $s$-perimeter in any compact set of $\mathbb{R}^{2}$, then it is necessarily a halfplane. Moreover, Theorem 1.3 extends this classification result to the class of stable sets.

In Corollary 1.21 we will obtain also results in dimension $n=3$ for minimizers of anisotropic interactions with a finite range of dependence (i.e. for "truncated kernels").

The proofs of our main results have, as starting point, a nontrivial refinement of the variational argument introduced by Savin and one of the authors in [37, 38] to prove that halfplanes are the only cones minimizing the $s$-fractional perimeter in every compact set of $\mathbb{R}^{2}$. Namely, we consider perturbations $E_{R, t}$ of a minimizer $E$ which coincide with $E$ outside $B_{R}$ and are translations $E+t \boldsymbol{v}$ of $E$ in $B_{R / 2}$ - with "infinitesimal" $t>0$. A first step in the proof is estimating how much $P_{K, B_{R}}\left(E_{R, t}\right)$ differs from $P_{K, B_{R}}(E)$ depending on $R$ - this is done in Lemma 2.1. By exploiting the nonlocality of the perimeter functional, the previous control on $P_{K, B_{R}}\left(E_{R, t}\right)-$ $P_{K, B_{R}}(E)$ is translated into a control on the minimum between $\left|E_{R, t} \backslash E\right|$ and $\left|E \backslash E_{R, t}\right|$ - the crucial estimates for this are given in Lemmas 2.2 and 2.4. Then, a careful geometric analysis allows us to deduce our main results - i.e. Theorems 1.7, 1.9. 1.14, 1.19 and their corollaries. We emphasize that we always use arbitrarily small perturbations of our set $E$. That is why we can establish some results for stable sets.

In the following subsections, we introduce the mathematical framework of nonlocal perimeters, we discuss some motivations for this general framework, and we present the main results obtained. 
1.1. The mathematical framework of nonlocal perimeter functionals. The notion of fractional perimeter was introduced in [11]. Let $s \in(0,1)$. Given a bounded domain $\Omega \subset \mathbb{R}^{n}$, we define the fractional $s$-perimeter of a measurable set $E \subset \mathbb{R}^{n}$ relative to $\Omega$ as

$$
P_{s, \Omega}(E):=L_{s}(E \cap \Omega, \mathcal{C} E \cap \Omega)+L_{s}(E \cap \Omega, \mathcal{C} E \backslash \Omega)+L_{s}(E \backslash \Omega, \mathcal{C} E \cap \Omega),
$$

where $\mathcal{C} E$ denotes the complement of $E$ in $\mathbb{R}^{n}$ and the interaction $L_{s}$ of two disjoint measurable sets $A, B$ is defined by

$$
L_{s}(A, B):=\int_{A} \int_{B} \frac{d x d \bar{x}}{|x-\bar{x}|^{n+s}} .
$$

Roughly speaking, this $s$-perimeter captures the interactions between a set $E$ and its complement. These interactions occur in the whole of the space and are weighted by a (homogeneous and rotationally invariant) kernel with polynomial decay (see Figure 1). Here, the role of the domain $\Omega$ is to "select" the contributions which arise in a given portion of the space and to "remove" possible infinite contributions to the energy which come from infinity but which do not change the variational problem.
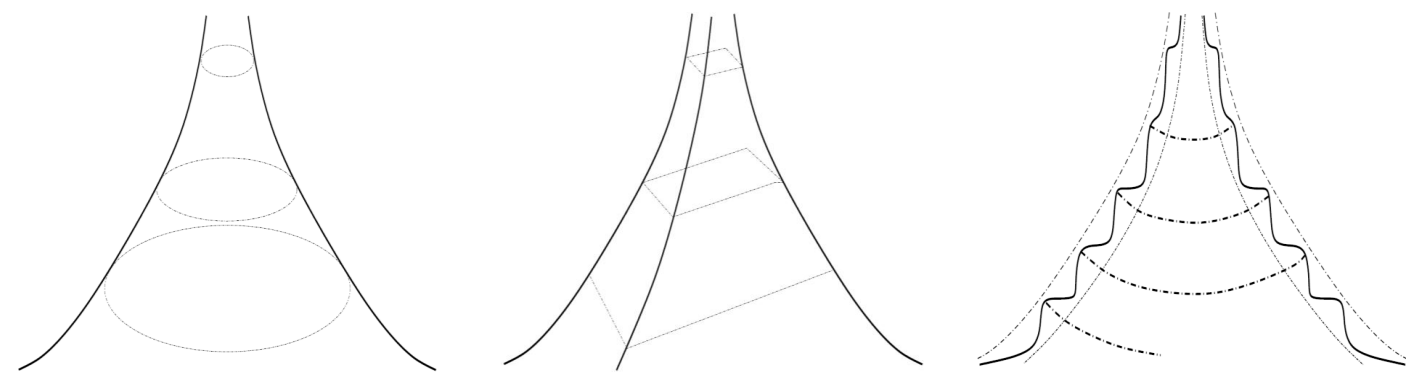

FiguRE 1. Kernels for: the $s$-perimeter, the anisotropic $s$-perimeter, more general $\mathcal{L}_{2}$ kernels.

A set $E$ is said to minimize the $s$-perimeter in $\Omega$ if

$$
P_{s, \Omega}(E) \leqslant P_{s, \Omega}(F), \quad \text { for all } F \text { with } E \backslash \Omega=F \backslash \Omega \text {. }
$$

The (boundaries of the) minimizers of the $s$-perimeter are often called nonlocal minimal (or $s$-minimal) surfaces.

In this paper, we study a more general functional, in which the interaction kernel is not necessarily homogeneous and rotational invariant. We consider a kernel $K$ satisfying

$$
\begin{gathered}
K(z) \geqslant 0 \\
K(z)=K(-z), \\
\int_{\mathbb{R}^{n}} K(z) \min \{1,|z|\} d z<+\infty
\end{gathered}
$$


and

$$
K \geqslant 1 \quad \text { in } B_{2} \text {. }
$$

To prove our main results we will require the following additional assumption on the first and second derivatives of the kernel $K$ :

$$
\max \left\{|z|\left|\partial_{e} K(z)\right|,|z|^{2} \sup _{|y-z| \leqslant|z| / 2}\left|\partial_{e e} K(y)\right|\right\} \leqslant K^{*}(z)
$$

for all $z \in \mathbb{R}^{n} \backslash\{0\}$ and for all $e \in S^{n-1}$, for some kernel $K^{*}$.

Throughout the paper we will have one of the three following cases:

- $K^{*}(z)=C_{1} K(z)$;

- $K^{*}(z)=C_{1}\left(K(z)+\chi_{\left\{|z|<R_{0}\right\}}(z)\right)$ for some $R_{0} \geqslant 2$;

- $K^{*}(z) \in L^{1}\left(\mathbb{R}^{n}\right)$.

We emphasize that the kernels of the fractional $s$-perimeter and its anisotropic version satisfy (1.7) with $K^{*}(z)=C_{1} K(z)$. Therefore, a reader interested in the results for these particular cases, can mentally replace $K^{*}$ by $C_{1} K$ in all the paper. We allow the second case of $K^{*}$ in order to obtain results for compactly supported kernels, as for example $\left(9-|z|^{2}\right)_{+}^{3}|z|^{-n-s}$. With the third case, we will be able to obtain strong results for nonsingular kernels like $e^{9-|z|^{2}}$.

We set

$$
L_{K}(A, B)=\int_{A} \int_{B} K(x-\bar{x}) d x d \bar{x} .
$$

We define, for a measurable set $E \subset \mathbb{R}^{n}$, the $K$-perimeter of $E$ in $\mathbb{R}^{n}$ as

$$
P_{K}(E)=L_{K}(E, \mathcal{C} E) \text {. }
$$

We define the $K$-perimeter of $E$ inside $\Omega, P_{K, \Omega}(E)$ similarly as in (1.1) with $L_{K}$ replacing $L_{s}$. That is,

$$
P_{K, \Omega}(E):=L_{K}(E \cap \Omega, \mathcal{C} E \cap \Omega)+L_{K}(E \cap \Omega, \mathcal{C} E \backslash \Omega)+L_{K}(E \backslash \Omega, \mathcal{C} E \cap \Omega)
$$

Note that our definition of $P_{K, \Omega}(E)$ agrees with the one of $P_{K}(E, \Omega)$ given in [24, Section 3].

Remark 1.4. We observe that if $K$ satisfies (1.5), then every Lipschitz bounded domain $U$ has finite $K$-perimeter in $\mathbb{R}^{n}$. Indeed,

$$
\begin{aligned}
P_{K}(U) & =\int_{U} \int_{\mathcal{C} U} K(\bar{x}-x) d x d \bar{x}=\int_{\mathbb{R}^{n}} d z \int_{U \cap(\mathcal{C} U-z)} d x K(z) \\
& =\int_{\mathbb{R}^{n}}|U \backslash(U-z)| K(z) d z \leqslant C \int_{\mathbb{R}^{n}} \min \{1,|z|\} K(z) d z<\infty
\end{aligned}
$$

where we have used the change of variables $z=\bar{x}-x$ and Fubini Theorem.

We next formally state the definition of minimizer of the $K$-perimeter. 
Definition 1.5. We say that $E$ is a minimizer for $P_{K, \Omega}$ in an open bounded set $\Omega$, if $P_{K, \Omega}(E)<\infty$ and

$$
P_{K, \Omega}(E) \leqslant P_{K, \Omega}(F)
$$

for any set $F$ which coincides with $E$ outside $\Omega$, that is $F \backslash \Omega=E \backslash \Omega$.

We also define the notion of stable set for the $K$-perimeter.

Definition 1.6. We say that $E$ is a stable set for $P_{K, \Omega}$ if $P_{K, \Omega}(E)<\infty$ and for any given vector field $X=X(x, t) \in C_{c}^{2}\left(\Omega \times(-1,1) ; \mathbb{R}^{n}\right)$ and $\varepsilon>0$ there is $t_{0}>0$ such that the following holds. Denoting $F_{t}=\Psi_{t}(E)$, where $\Psi_{t}$ is the integral flow of $X$, we have

$$
0 \leqslant P_{K, \Omega}\left(F_{t} \cup E\right)-P_{K, \Omega}(E)+\varepsilon t^{2}
$$

and

$$
0 \leqslant P_{K, \Omega}\left(F_{t} \cap E\right)-P_{K, \Omega}(E)+\varepsilon t^{2}
$$

for all $t \in\left(-t_{0}, t_{0}\right)$.

For our second theorem we will consider kernels $K$ in the class $\mathcal{L}_{2}(s, \lambda, \Lambda)$ introduced by Caffarelli and Silvestre in [12] (see Figure 1). Namely, the kernels $K(z)$ satisfying (1.4),

$$
\frac{\lambda}{|z|^{n+s}} \leqslant K(z) \leqslant \frac{\Lambda}{|z|^{n+s}}
$$

and

$$
\max \left\{|z|\left|\partial_{e} K(z)\right|,|z|^{2}\left|\partial_{e e} K(z)\right|\right\} \leqslant \frac{\Lambda}{|z|^{n+s}}
$$

for all $z \in \mathbb{R}^{n} \backslash\{0\}$ and for all $e \in S^{n-1}$. Note that, after multiplying a kernel $K \in \mathcal{L}_{2}$ by a positive constant, we may assume that $\lambda \geqslant 2^{n+s}$ and hence $K$ satisfies (1.3) 1.7 with $K^{*}=C_{1} K$.

A very relevant particular case to which our results apply is that of $s$-fractional anisotropic perimeters, introduced in [30]. This case corresponds to the choice of the kernel

$$
K(z)=\frac{a(z /|z|)}{|z|^{n}}
$$

where $a$ is some positive, even $C^{2}$ function on the $(n-1)$-dimensional unit sphere $S^{n-1}$ (see Figure 1). The notion of anisotropic nonlocal perimeter was considered in [30], where some asymptotic results for $s \rightarrow 1^{-}$where established.

1.2. Motivations of nonlocal perimeters. To favor a concrete intuition of the nonlocal perimeter functional, we now recall some practical applications of the nonlocal perimeter functionals. In these applications, it is also natural to consider interactions that are not homogeneous or rotationally invariant.

A. The first application that we present is related to image processing and bitmaps. 
Let us consider the framework of BMP type images with square pixels of (small) size $\rho>0$ (and suppose that $1 / \rho \in \mathbb{N}$ for simplicity). For simplicity, let us consider a picture of a square of side 1 , with sides at $45^{\circ}$ with respect to the orientation of the pixels and let us compare with the "version" of the square which is represented in the image (see Figure 2).

In this configuration, the classical perimeter functional provides a rather inaccurate tool to analyze this picture, no matter how small the pixels are, i.e. no matter how good is the image resolution.

Indeed, the perimeter of the ideal square is 4 , while the perimeter of the picture displayed by the monitor is always $4 \sqrt{2}$ (independently on the smallness of $\rho$ ), so the classical perimeter is always producing an error by a factor $\sqrt{2}$, even in cases of extremely high resolution.

Instead, the fractional perimeter (for instance with $s=0.95$ ) or other nonlocal perimeters would provide a much better approximation of the classical perimeter of the ideal square in the case of high image resolution. Indeed, the discrepancy $D_{s}(\rho)$ between the $s$-perimeter of the ideal square and the $s$-perimeter of the pixelled square is bounded by above by the sum of the interactions between the "boundary pixels" with their complement: these pixels are the ones which intersect the boundary of the original square, and their number is $4 / \rho$.

By scaling, the interaction of one pixel with its complement is of the order of $\rho^{2-s}$, therefore we obtain that $D_{s}(\rho) \leqslant C \rho^{1-s}$, which is infinitesimal as $\rho \rightarrow 0$.

Since the fractional perimeter (suitably normalized) is close to the classical one as $s \rightarrow 1^{-}$, that the fractional perimeter provides in this case a more precise information that the classical one.

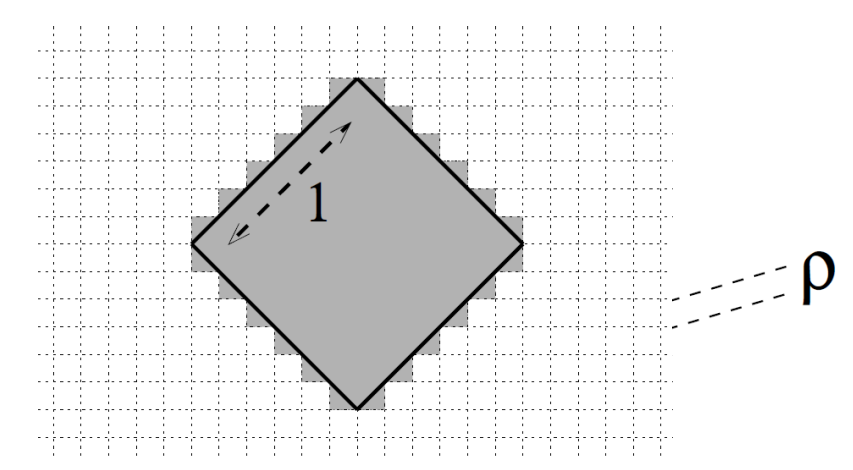

Figure 2. Discrepancy of local/nonlocal perimeters in a bitmap.

B. Another main motivation for the study of nonlocal $s$-minimal surfaces, as explained in [11, is the understanding of steady states for nonlinear interface evolution processes with Lévy diffusion. Namely let us think of $u(t, \cdot): \mathbb{R}^{n} \rightarrow[0,1]$ as representing the state at time $t$ of some interface phenomenon where two stable states 
$u \equiv 1$ and $u \equiv 0$ diffuse and "compete" to conquer the whole space. In concrete applications $u$ could be, for instance, the density of an invasive biological specie.

For a wide class of such situations, the evolution equation that governs $u$ is of the type

$$
u_{t}+\mathcal{L} u=f(u)
$$

where $\mathcal{L}$ is a "diffusion operator" - e.g. $\mathcal{L}=(-\Delta)^{s / 2}, s \in(0,2]$ - and $f$ is a bistable nonlinearity with $f(0)=f(1)=0$ and $f(z)$ increasing (resp. decreasing) near $z=0$ (resp. $z=1$ ).

An extreme version of this evolution process, heuristically corresponding to a huge balanced $f$ like $f(u)=M\left((2 u-1)-(2 u-1)^{3}\right)$ with $M \gg 1$ is the following.

Given an open set $E \subset \mathbb{R}^{n}$ with smooth boundary we define its density function $u$ by

$$
u(x)=\lim _{r \searrow 0} \frac{\left|B_{r}(x) \cap E\right|}{\left|B_{r}(x)\right|} .
$$

That is $u(x)$ takes the values $1,1 / 2$ or 0 depending on whether $x$ belongs to $E, \partial E$ or the interior of $\mathcal{C} E$

Let $\mathcal{L}$ be a "diffusion operator", or more rigorously, an infinitesimal generator of a Lévy process. For $t \in \tau \mathbb{N} \cup\{0\}$, where $\tau$ is a tiny time step, we define the discrete in time evolution $\Phi_{t}^{\mathcal{L}}(u)$ of the density function $u$ of $E$ as follows:

$$
\Phi_{t+\tau}^{\mathcal{L}}(u)(x)= \begin{cases}1 & \text { if } v(\omega, x)>1 / 2 \\ 1 / 2 & \text { if } v(\omega, x)=1 / 2 \\ 0 & \text { if } v(\omega, x)<1 / 2,\end{cases}
$$

where $\omega=\omega(\tau)$ is an appropriate time step depending on $\tau$ and $v$ is the solution to

$$
v_{t}+\mathcal{L} v=0 \quad \text { with initial condition } v(0, \cdot)=\Phi_{t}^{\mathcal{L}}(u) .
$$

In this way $\Phi_{t}^{\mathcal{L}}$ defines a discrete in time surface evolution of $\partial E$ - excluding patological cases in which thickening of the set $\{v=1 / 2\}$ might occur.

Heuristically, a set $E$ with smooth enough boundary will be stationary under the flow $\Phi_{t}^{\mathcal{L}}$ (with infinitesimal $\tau$ ) if and only if its density function $u$ satisfies

$$
\mathcal{L} u(x)=0 \quad \text { for all } x \in \partial E=\{u=1 / 2\} .
$$

Indeed, in this way the evolution $v_{t}+\mathcal{L} v=0$ will be $v_{t} \approx 0$ on $\partial E$ for $0<t<$ $\omega(\tau) \ll 1$, and the boundary points will not move. Note that this heuristic argument is independent of the modulus of continuity $\omega$.

In some cases, under an appropriate choice of $\omega=\omega(\tau)$ the discrete flow $\Phi_{t}^{\mathcal{L}}$ can be shown to converge to some continuous flow as $\tau \searrow 0$.

When $\mathcal{L}=-\Delta$ is the Laplacian, under the choice $\omega=\tau$ the $\Phi_{t}^{\mathcal{L}}$ converges to the mean curvature flow. This classical result was conjectured by Merriman, Bence, and Osher in [33], and proven to be true by Evans [22] and Barles and Georgelin [3]. In [17], Chambolle and Novaga generalized this result to the case of anisotropic and crystalline curvature motion. In [29] Ishii, Pires and Souganidis 
study the convergence of general threshold dynamics type approximation schemes to hypersurfaces moving with normal velocity depending on the normal direction and the curvature tensor.

Finally, in [13], the case $\mathcal{L}=(-\Delta)^{s / 2}$ was considered: in this case $\Phi_{t}^{\mathcal{L}}$ still converges to the mean curvature flow for $s \in(1,2)$ with $\omega=\tau^{s / 2}$ and for $s=1$ with $\omega$ implicitly defined $\omega^{2}|\log \omega|=\tau$ for $\tau$ small.

Instead, for $s \in(0,1)$ and $\omega=\tau^{s /(1+s)}$, the discrete flow $\Phi_{t}^{\mathcal{L}}$ with $\mathcal{L}=(-\Delta)^{s / 2}$ converges to a new geometric flow: the $s$-nonlocal mean curvature flow (see [13], Theorem 1) - a flow where the normal displacement is proportional to the nonlocal mean curvature; see also [16, 28, 36]. Fractional $s$-minimal surfaces are stationary under this $s$-nonlocal mean curvature flow.

At the level of discrete flow, we can replace $(-\Delta)^{s / 2}$ with a more general elliptic operator of form

$$
\mathcal{L} u(x)=\int_{\mathbb{R}^{n}}(u(x)-u(\bar{x})) K(x-\bar{x}) d \bar{x}
$$

where $K$ satisfies (1.3)-(1.6). Heuristically, minimizers of the $K$-perimeter should be natural candidates to being stationary under the flow $\Phi_{t}^{\mathcal{L}}$ as $\tau \rightarrow 0$.

C. Another motivation for the study of nonlocal $s$-minimal surfaces comes from models describing phase-transitions problems with long-range interactions. In the classical theory of phase transitions, one consider the energy functional

$$
\mathcal{E}(u)=\int_{\Omega} \varepsilon^{2}|\nabla u|^{2}+W(u),
$$

where $W$ is a double well potential representing the dislocation energy, and the first term, involving $\nabla u$, penalizes the formation of unnecessary interfaces. The classical $\Gamma$-convergence result by Modica and Mortola [34] states that the energy functional $\varepsilon^{-1} \mathcal{E} \Gamma$-converges to the (classical) perimeter functional. A nonlocal analogue of 1.14 is the following

$$
\mathcal{E}_{\sigma}(u)=\varepsilon^{2 \sigma} \mathcal{K}_{\sigma}(u, \Omega)+\int_{\Omega} W(u)
$$

where

$$
\mathcal{K}_{\sigma}(u, \Omega):=\frac{1}{2} \int_{\Omega} \int_{\Omega} \frac{|u(x)-u(\bar{x})|^{2}}{|x-\bar{x}|^{n+2 \sigma}} d x d \bar{x}+\int_{\Omega} \int_{\mathcal{C} \Omega} \frac{|u(x)-u(\bar{x})|^{2}}{|x-\bar{x}|^{n+2 \sigma}} d x d \bar{x} .
$$

The previous energy functional models long range (or nonlocal) interactions between the particles - the density of particles at a point is influenced by the density at other points that may be not infinitesimally close. The minimizers of the functional $\mathcal{E}_{\sigma}(u)$ have been studied in several recent papers [6, 7, 10, 8, 9, 42. A list of results established in these works includes: 1-D symmetry in low dimensions, energy estimates, Hamiltonian identities, existence and decay properties of 1-D solutions, etc. 
In [39, Savin and one of the authors study the $\Gamma$-convergence of the energy functional $\mathcal{E}_{\sigma}$. In particular, they prove that when $\sigma \in[1 / 2,1)$, after a suitable rescaling, $\mathcal{E}_{\sigma} \Gamma$-converges to the classical perimeter functional. On the other hand, when $\sigma \in(0,1 / 2)$ the functional $\varepsilon^{-2 \sigma} \mathcal{E}_{\sigma} \Gamma$-converges to the nonlocal $s$-perimeter with $s=2 \sigma$. Note that $\varepsilon^{-2 \sigma} \mathcal{E}_{\sigma}=\mathcal{K}_{\sigma}+\varepsilon^{-2 \sigma} \int_{\Omega} W(u)$ and thus, in this renormalization, there is no small coefficients in front of the Dirichlet energy.

Analogously, we could consider more energy functionals of the form

$$
\begin{aligned}
\mathcal{E}_{K}(u)= & \frac{1}{2} \int_{\Omega} \int_{\Omega}|u(x)-u(\bar{x})|^{2} K(x-\bar{x}) d x d \bar{x} \\
& +\int_{\Omega} \int_{\mathcal{C} \Omega}|u(x)-u(\bar{x})|^{2} K(x-\bar{x}) d x d \bar{x}+M \int_{\Omega} W(u),
\end{aligned}
$$

where $M \gg 1$ is a large real number.

Heuristically, similarly to the result of [39], minimizers of $\mathcal{E}_{K}$ should "converge" to minimizers of the $K$-perimeter $P_{K}$ as $M \rightarrow \infty$.

1.3. Statement of the main results. From now on, we will assume that $K$ satisfies assumptions (1.3)-(1.7).

We state our main results in the two following subsections. In the first one we give the uniform $B V$-estimates for stable sets, and their consequence on existence and compactness of minimizers of the $K$-perimeter - see [23, 27, 31]. In the second one we state our quantitative flatness results and we comment on some corollaries and some applications for specific choices of the kernels that are of independent interest.

1.3.1. Uniform $B V$-estimates. We recall the (classical) notion of $B V$-space and of sets of finite perimeter. Let $\Omega$ be an open set of $\mathbb{R}^{n}$. Given a function $u$ in $L^{1}(\Omega)$, the total variation of $u$ in $\Omega$ is defined as follows:

$$
|\nabla u|(\Omega):=\sup \left\{\int_{\Omega} u \operatorname{div} \phi \text { with } \phi \in C_{c}^{1}\left(\Omega, \mathbb{R}^{n}\right),|\phi| \leqslant 1\right\} .
$$

Here, and throughout the paper, we denote $C_{c}^{1}(U ; A)$ the $C^{1}$ vector fields compactly supported in $U$ and taking values in $A$.

The space $B V(\Omega)$ is defined as the space of functions which belong to $L^{1}(\Omega)$ and have $|\nabla u|(\Omega)$ finite. Moreover, we say that a set $E \subset \mathbb{R}^{n}$ has finite perimeter in $\Omega$, when the distributional gradient $\nabla \chi_{E}$ of its characteristic function is a $\mathbb{R}^{n}$-valued Radon measure on $\mathbb{R}^{n}$ and $\left|\nabla \chi_{E}\right|(\Omega)<\infty$. In this case, we define the perimeter of $E$ in $\Omega$ as:

$$
\operatorname{Per}_{\Omega}(E)=\left|\nabla \chi_{E}\right|(\Omega)
$$

Finally, we define the reduced boundary $\partial^{*} E$ of a set of finite perimeter $E$ as follows: $\partial^{*} E$ is the set of all points $x$ such that $\left|\nabla \chi_{E}\right|\left(B_{r}(x)\right)>0$ for any $r>0$ and

$$
\lim _{r \rightarrow 0^{+}} \frac{\nabla \chi_{E}\left(B_{r}(x)\right)}{\left|\nabla \chi_{E}\left(B_{r}(x)\right)\right|} \quad \text { exists and belongs to } S^{n-1} .
$$


For any $x \in \partial^{*} E$, we denote by $-\nu_{E}(x)$ the limit in 1.15 and we call the Borel vector field $\nu_{E}: \partial^{*} E \rightarrow S^{n-1}$ the measure theoretic outer unit normal to $E$.

The following are our main results:

Theorem 1.7 ( $B V$-estimates for stable sets). Let $n \geqslant 2$. Let $E$ be a stable set of the $K$-perimeter in $B_{4}$, with $K$ in $\mathcal{L}_{2}(s, \lambda, \Lambda)$, that is, with $K$ satisfying (1.4), (1.9), and (1.10).

Then, the classical perimeter of $E$ in $B_{1}$ is finite. Namely $\chi_{E}$ belongs to $B V\left(B_{1}\right)$ with the following universal estimate

$$
\operatorname{Per}_{B_{1}}(E)=\left|\nabla \chi_{E}\right|\left(B_{1}\right) \leqslant C(n, s, \lambda, \Lambda) .
$$

Rescaling Theorem 1.7, and using an interpolation inequality that relates $P_{K}$ and Per, we obtain

Corollary 1.8. Let $n \geqslant 2$. Let $E$ be stable set of the $K$-perimeter in $B_{4 R}$, with $K$ in $\mathcal{L}_{2}(s, \lambda, \Lambda)$, i.e. satisfying condition (1.4), (1.9) and (1.10). Then,

$$
\operatorname{Per}_{B_{R}}(E) \leqslant C(n, s, \lambda, \Lambda) R^{n-1} \text {. }
$$

As a consequence

$$
P_{K, B_{R}}(E) \leqslant C(n, s, \lambda, \Lambda) R^{n-s} .
$$

We observe that the exponent $n-s$ in 1.17 is optimal since it is achieved when $E$ is an halfspace. To prove (1.17) when $E$ is minimizer, it is enough to compare the $K$-perimeter of $E$ with the $K$-perimeter of $E \cup B_{R}$. However, for stable stationary sets this simple comparison argument can not be done and the proof is much more involved - we need to prove our (stronger) uniform $B V$-estimates and deduce (1.17) as a byproduct.

Theorem 1.7 follows from the following result for general kernels combined with an appropriate scaling and covering argument.

Theorem 1.9. Let $n \geqslant 2$. Let $E$ be a stable set of the $K$-perimeter in $B_{4}$, with $K$ satisfying $(1.3)-(1.7)$. If $P_{K^{*}, B_{4}}(E)<\infty$, then the classical perimeter of $E$ in $B_{1}$ is finite. Namely $\chi_{E}$ belongs to $B V\left(B_{1}\right)$ with the following estimate

$$
\operatorname{Per}_{B_{1}}(E)=\left|\nabla \chi_{E}\right|\left(B_{1}\right) \leqslant \sqrt{2} n \sqrt{P_{K^{*}, B_{4}}(E)}+\left|S^{n-1}\right| .
$$

Here, $\left|S^{n-1}\right|$ denotes the $(n-1)$-dimensional measure of the sphere $S^{n-1}$.

Theorem 1.9 can be applied to several particular cases. We state below the ones which we consider more relevant.

Corollary 1.10. Let $n \geqslant 2$. Let $E$ be a stable set of the $K$-perimeter in $B_{4}$, with $K$ satisfying $(1.3)-(1.7)$ and $K^{*} \in L^{1}\left(\mathbb{R}^{n}\right)$. Then,

$$
\operatorname{Per}_{B_{1}}(E)=\left|\nabla \chi_{E}\right|\left(B_{1}\right) \leqslant\left.\sqrt{2} n\left|B_{4}\right|^{1 / 2}|| K^{*}\right|_{L^{1}\left(\mathbb{R}^{n}\right)} ^{1 / 2}+\left|S^{n-1}\right| .
$$


Recall that we denote the $K$-perimeter of a ball $B_{R}$ (relative to $\mathbb{R}^{n}$ ) as

$$
P_{K}\left(B_{R}\right)=\int_{B_{R}} \int_{\mathcal{C} B_{R}} K(\bar{x}-x) d \bar{x} d x .
$$

We remark that for kernels as in 1.11 we have that $P_{K}\left(B_{R}\right)=C R^{n-s}$. Notice also that, by a simple comparison argument,

$$
\sup \left\{P_{K, B_{R}}(E): E \text { minimizer of the } K \text {-perimeter in } B_{R}\right\} \leqslant P_{K}\left(B_{R}\right) \text {. }
$$

Indeed, if $E$ is a minimizer in $B_{R}$, then $P_{K, B_{R}}(E) \leqslant P_{K, B_{R}}\left(E \cup B_{R}\right) \leqslant P_{K}\left(B_{R}\right)$.

When $E$ is a minimizer and $K^{*}=C_{1}\left(K+\chi_{|z|<R_{0}}\right)$, then $P_{K^{*}, B_{R}}(E)$ can be bounded by above by $C P_{K}\left(B_{R}\right)$. This is the content of the following proposition (which is proven later on in Section 5).

Proposition 1.11. Let $E$ be a minimizer of the $K$-perimeter in $B_{R}$ with $R \geqslant 1$ and $K$ satisfying (1.3) - (1.7), and $K^{*}=C_{1}\left(K+\chi_{|z|<R_{0}}\right)$ for some $R_{0} \geqslant 2$.

Then,

$$
P_{K^{*}, B_{R}}(E) \leqslant C C_{1} P_{K}\left(B_{R}\right)
$$

where $C$ is a constant depending only on $n$ and $R_{0}$.

As a consequence of Theorem 1.9 and Proposition 1.11, we deduce the following

Corollary 1.12 ( $B V$-estimates for minimizers). Let $E$ be a minimizer of the $K$-perimeter in $B_{4}$, with $K$ satisfying $(1.3)-(1.7)$, and $K^{*}=C_{1}\left(K+\chi_{|z|<R_{0}}\right)$ for some $R_{0} \geqslant 2$.

Then,

$$
\operatorname{Per}_{B_{1}}(E)=\left|\nabla \chi_{E}\right|\left(B_{1}\right) \leqslant C \sqrt{C_{1} P_{K}\left(B_{4}\right)}
$$

where $C$ is a constant depending only on $n$ and $R_{0}$.

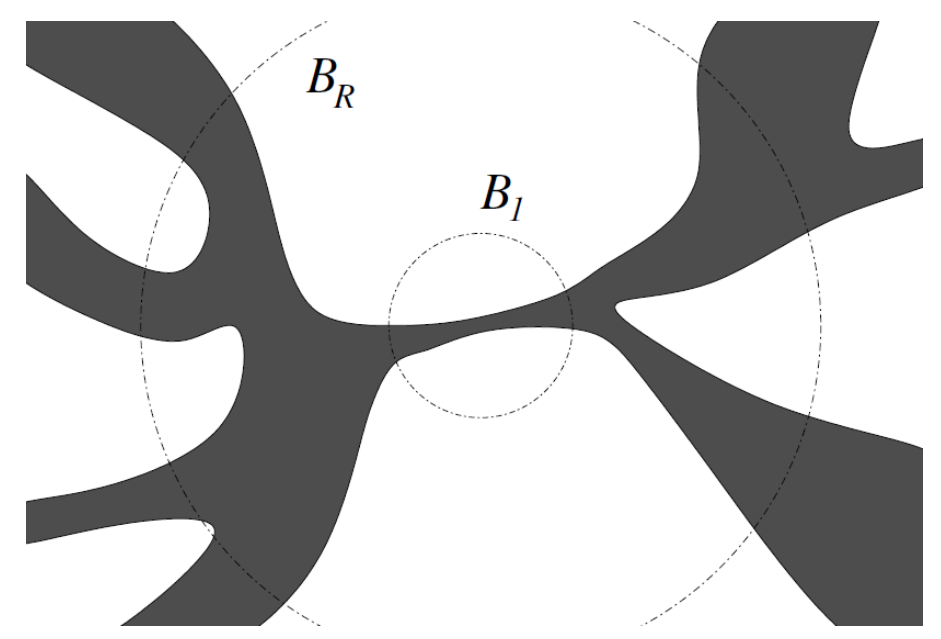

Figure 3. A minimizer of the $K$-perimeter in $B_{R}$ has finite classical perimeter in $B_{1}$. 
As explained in the beginning of the introduction, the "a priori" $B V$-estimate established in Corollary 1.12 allows us to prove a very general existence result for minimizers of $P_{K, \Omega}$. We state it next.

Theorem 1.13 (Existence of minimizers). Let $\Omega$ be a bounded Lipschitz domain, and $E_{0} \subset \mathcal{C} \Omega$ a given measurable set. Suppose that $K$ satisfies assumptions (1.3)(1.7). Then, there exists a set $E$, with $E \cap \mathcal{C} \Omega=E_{0}$ that is a minimizer for $P_{K, \Omega}$ -in particular $P_{K, \Omega}(E)<\infty$.

Notice that when $K$ belongs to $L^{1}\left(\mathbb{R}^{n}\right)$, then $P_{K, \Omega}(F)<|\Omega| \int_{\mathbb{R}_{n}} K<\infty$ for all measurable sets $F$. Thus, in principle, we do not have compactness for sequences of sets with uniformly bounded $K$-perimeter.

The idea of the proof of Theorem 1.13 (which will be given in Section 4) consists in considering the "singularized" kernel

$$
K_{\varepsilon}(z):=K(z)+\frac{\varepsilon}{|z|^{n+\frac{1}{2}}},
$$

which, for every fixed $\varepsilon$, admits a minimizer $E_{\varepsilon}$ by the standard compactness of $H^{\frac{1}{4}}$ in $L^{1}$. All the new kernels $K_{\varepsilon}$ satisfy assumptions $(1.3)-(1.7)$ with constants that are uniform in $\varepsilon$. Thus, Theorem 1.12 gives uniform $B V$-bounds for the characteristic functions of the minimizers $E_{\varepsilon}$. These bounds give the necessary compactness in $L^{1}$ to prove the existence of a limiting set as $\varepsilon \rightarrow 0$. In order to prove that the limiting set is a minimizer of $P_{K, \Omega}$, we use some other important ingredients (such as a nonlocal coarea formula and a density result for smooth sets into sets of finite $K$-perimeter) that will be established later on in Section 6 .

1.3.2. Quantitative flatness results. Our quantitative flatness results in low dimensions $n=2,3$ state that (under appropriate assumptions on the kernel $K$ ) a stable set $E$ of the $K$-perimeter in a very large ball $B_{R}$ is close to being a flat graph in $B_{1}$. Namely, for some $\varepsilon=\varepsilon(R)$ that decreases to 0 when $R$ increases to $\infty$, and after a rotation of coordinates, the following three properties hold.

(F1) For some $t \in[-1,1]$,

$$
\left|\left(E \triangle\left\{x_{n} \leqslant t\right\}\right) \cap B_{1}\right| \leqslant \varepsilon,
$$

where $\triangle$ denotes the symmetric difference.

(F2) There is a set $\mathcal{B} \subset B_{1}^{(n-1)}=\left\{x^{\prime} \in \mathbb{R}^{n-1}:\left|x^{\prime}\right| \leqslant 1\right\}$ with $|\mathcal{B}| \leqslant \varepsilon$ such that $\left(E \cap B_{1}\right) \backslash(\mathcal{B} \times \mathbb{R})=\left\{\left(y, x_{n}\right) \in B_{1}: x_{n} \leqslant g(y), y \in\left(B_{1}^{(n-1)} \backslash \mathcal{B}\right)\right\}$.

for some measurable function $g: B_{1}^{(n-1)} \rightarrow[-1,1]$.

(F3) Denoting $F^{\varepsilon}=\left\{\left(x^{\prime}, x_{n} / \varepsilon\right):\left(x^{\prime}, x_{n}\right) \in F\right\}$, we have

$$
\operatorname{Per}_{B_{1}^{\varepsilon}}\left(E^{\varepsilon}\right) \leqslant C(n)
$$
where $C(n)$ is a constant depending only on the dimension $n \in\{2,3\}$. 
Point (F1) says that the set $E$ is close in the $L^{1}$-sense to being a half-plane while point (F2) says that $\partial E \cap B_{1}$ is a graph after removing "vertical" cylinders of small measure (see Figure 4). Moreover, (F3) gives a uniform bound for the classical perimeter of rescalings of $E$ in the vertical direction by a large factor $1 / \varepsilon$.
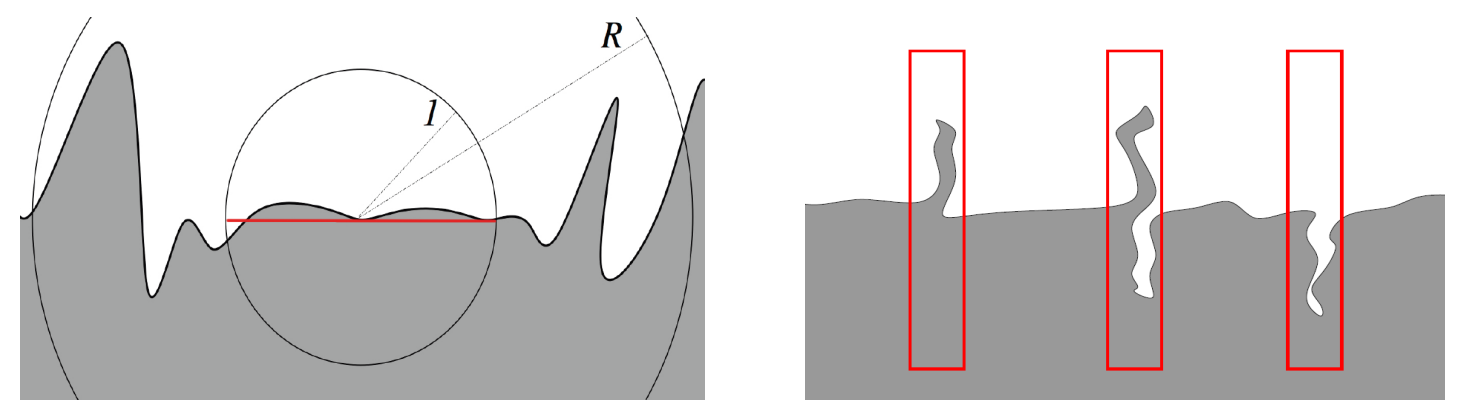

Figure 4. A minimizer or stable set of the $K$-perimeter in $B_{R}$ is "almost" a flat graph in $B_{1}$.

We give below our quantitative flatness result for stable sets in dimension $n=2$.

Theorem 1.14 (Flatness for stable sets in dimension two). Let $n=2$. Let $K$ be a kernel belonging to the class $\mathcal{L}_{2}(s, \lambda, \Lambda)$, i.e. satisfying conditions (1.4), (1.9), and $(1.10)$. Let $E$ is a stable set of the $K$-perimeter in $B_{R}$ with $R \geqslant 4$.

Then, after a rotation, E satisfies $(\mathbf{F} 1),(\mathbf{F} 2)$, and $(\mathbf{F} 3)$ with

$$
\varepsilon=C R^{-s / 2}
$$

where $C$ is a constant depending only on $s, n, \lambda, \Lambda$.

Remark 1.15. We recall that Theorem 1.14 applies, in particular, to the fractional anisotropic perimeter introduced in [30], where

$$
K(z)=\frac{a(z /|z|)}{|z|^{n+s}}
$$

with $a \in C^{2}\left(S^{n-1}\right)$ positive.

For the sake of clarity, let us rephrase the first conclusion of Theorem 1.14 in the following way: Let $K \in \mathcal{L}_{2}$ and let $E$ be a stable set of $P_{K, B_{R}}$. Then, there exists a halfplane $\mathfrak{h}$ such that

$$
\left|(E \triangle \mathfrak{h}) \cap B_{1}\right| \leqslant C R^{-s / 2}
$$

Sending $R \rightarrow \infty$ in Theorem 1.14, we deduce the following

Corollary 1.16. For $K \in \mathcal{L}_{2}$, half-planes are the only stable sets in every compact set of $\mathbb{R}^{2}$. 
The local analogue of Corollary 1.16 was established in 20 , 26], where the following statement is proved: Any complete stable surfaces in $\mathbb{R}^{3}$ is a plane. As said above in the Introduction, this classification result for classical stable surfaces is still open in dimensions $n \geqslant 4$.

As explained previously in the Introduction, our quantitative flatness result for stable sets in Theorem 1.14 generalizes the classification theorem of [37], that we recall next.

Theorem 1.17. (Theorem 1 in [37]) Let $E$ be a cone that is a minimizer of $P_{s}$ in every compact set of $\mathbb{R}^{2}$. Then $E$ is a half-plane.

Using a blow-down argument and a monotonicity formula — see Remark 1.18 , Theorem 1.17 implies that halfplanes are the only minimizers of the $s$-perimeter in every compact set of $\mathbb{R}^{2}$.

Moreover, similarly as in the theory of classical minimal surfaces, this classification result has important consequences in the regularity theory for nonlocal $s$-minimal surfaces. In particular, combining Theorem 1.17 and the results contained in [4, 11], one can deduce that any minimizer of the $s$-perimeter is smooth outside of a singular set with Hausdorff dimension at most $n-3$.

Our Theorem 1.14 generalizes Theorem 1.17 in three directions. First, our result applies to the more general class of stable sets (we recall that any minimizer is a stable set). Second, we can consider more general kernels in $\mathcal{L}_{2}$. Third, our result is a quantitative version of Theorem 1.17 in the following sense: instead of assuming that $E$ is a minimizer in every compact set of $\mathbb{R}^{2}$, we assume that $E$ is a stable set for $P_{K, B_{R}}$ with some large $R$ and we obtain a quantitative control on the flatness of $E$ in $B_{1}$, depending on $R$.

We point out that, using the $C^{2}$ estimates for minimizers of the $s$-perimeter, and scaling invariance, the distance of $\partial E$ and some plane in $B_{1}$ is bounded by $C R^{-1}$, when $E$ is a minimizer of the $s$-perimeter in $B_{R}$. However, since the $C^{2}$ estimates are proved by compactness, we have no explicit estimates for this constant $C$. Moreover, such an approach clearly fails in case the problem is not scaling invariant or does not have a regularity theory. Note that with the techniques of this paper we can obtain results for general kernels that are not scaling invariant and for which the existence of some regularity theory is unclear — see for instance Corollary 1.20 .

Remark 1.18. We emphasize that for the specific case of $K(z)=|z|^{-n-s}$, Caffarelli, Roquejoffre and Savin proved a monotonicity formula for the local energy functional associated to the $s$-perimeter via the so called Caffarelli-Silvestre extension. This monotonicity formula allows them to use a blow-up argument to prove regularity results once one knows that the only nonlocal minimal cones are halfplanes.

On the other hand, as explained above, using the monotonicity formula and Theorem 1.17 one proves that halfplanes are the only minimizers of the $s$-perimeter in every compact set of $\mathbb{R}^{2}$ - thus extending the classification result from cones to all minimizers. 
In our setting, monotonicity formulas are not available but still we can obtain the same type of classification result as a consequence of our quantitative flatness estimates.

We will deduce Theorem 1.14 from the following more general result.

Theorem 1.19. Let $n=2,3$. Assume that $E$ is a stable set for the $K$-perimeter in $B_{R}$ with $R \geqslant 4$ and $K$ satisfying (1.3)-(1.7).

Then, after some rotation, $E$ satisfies $(\mathbf{F} \mathbf{1}),(\mathbf{F} 2)$, and $(\mathbf{F} 3)$ with

$$
\varepsilon=\varepsilon(R)=C \min \left\{\sqrt{\frac{P_{K^{*}, B_{R}}(E)}{R^{2}}}, \frac{1}{\sqrt{\log R}} \sup _{\rho \in[1, R]} \sqrt{\frac{P_{K^{*}, B_{\rho}}(E)}{\rho^{2}}}\right\},
$$

where $C$ is a constant depending only on $n$.

The result contained in Theorem 1.19 is very general and it can be applied to several choices of kernels. Below, we list some particular cases that are of independent interest.

For kernels with $K^{*} \in L^{1}\left(\mathbb{R}^{n}\right)$, we have the following

Corollary 1.20. Let $n=2$. Let $E$ be a stable set of the $K$-perimeter in $B_{R}$ with $R \geqslant 4$ and $K$ satisfying (1.3)-(1.7) with $K^{*} \in L^{1}\left(\mathbb{R}^{n}\right)$.

Then, after some rotation, E satisfies $(\mathbf{F 1}),(\mathbf{F} 2)$, and $(\mathbf{F} 3)$ with

$$
\varepsilon=\varepsilon(R)=\frac{C}{\sqrt{\log R}}\left|B_{1}\right|^{1 / 2}|| K^{*} \|_{L^{1}\left(\mathbb{R}^{n}\right)}^{1 / 2},
$$

where $C$ is a constant depending only on $n$.

Moreover, if $E$ is a minimizer for the $K$-perimeter, combining Theorem 1.19 and Proposition 1.11, we deduce

Corollary 1.21 (Flatness for minimizers in low dimensions). Let $n=2,3$. Let $E$ be a minimizer of the $K$-perimeter in $B_{R}$ with $R \geqslant 4$ and $K$ satisfying $(1.3)$ (1.7) with $K^{*}=C_{1}\left(K+\chi_{|z|<R_{0}}\right)$ for some $R_{0}>2$.

Then, after some rotation, E satisfies $(\mathbf{F 1}),(\mathbf{F} 2)$, and $(\mathbf{F} 3)$ with

$$
\varepsilon=\varepsilon(R)=C \min \left\{\sqrt{C_{1} \frac{P_{K}\left(B_{R}\right)}{R^{2}}}, \frac{1}{\log R} \sup _{\rho \in[1, R]} \sqrt{\frac{C_{1} P_{K}\left(B_{\rho}\right)}{\rho^{2}}}\right\},
$$

where $C$ is a constant depending only on $n$ and $R_{0}$.

Finally, as a particular case of Corollary 1.21, we consider the case of kernels with compact support.

Corollary 1.22 (Quantitative flatness for truncated kernels). Let $K$ satisfy (1.3)-1.7) and suppose that $K$ has compact support. Let $E$ be a minimizer of the $K$-perimeter in $B_{R}$ with $R \geqslant 4$. 
Then, after some rotation, E satisfies $(\mathbf{F} 1),(\mathbf{F} 2)$, and (F3) with

$$
\varepsilon= \begin{cases}C R^{-\frac{1}{2}} & \text { if } n=2, \\ \frac{C}{\sqrt{\log R}} & \text { if } n=3,\end{cases}
$$

where $C$ is a constant depending only on $n$ and $K$.

This result comes easily applying Corollary 1.21 and by the following energy estimate which holds for the case of a compactly supported kernel $K$ :

$$
P_{K}\left(B_{R}\right) \leqslant C R^{n-1} \text {. }
$$

As a consequence of Corollaries 1.20 and 1.22 , we obtain the following

Corollary 1.23. For kernels $K$ satisfying $(1.3)-(1.7)$, halfspaces are the only minimizers in every compact set of $\mathbb{R}^{n}$ in the following cases:

- $n=2$ and $K^{*} \in L^{1}\left(\mathbb{R}^{n}\right)$;

- $n=2,3$ and $K$ with compact support.

The paper is organized as follows:

- In section 2, we establish some preliminary results that we will use in the proof of our main theorems;

- In section 3, we prove Theorems 1.7 and 1.9 establishing the uniform $B V$ bounds for stable sets and for minimizers;

- In section 4, we prove our quantitative rigidity result (Theorems 1.14 and 1.19 ;

- Section 5 is dedicated to some technical lemmas that we need in the proofs of the main results;

- In section 6, we give the proof of the existence result (Theorem 1.13).

\section{Preliminary Results}

Following an idea in [37, we want to consider perturbations of the minimizer $E$ which are translations of $E$ in some direction $\boldsymbol{v}$ in $B_{R / 2}$ and coincide with $E$ outside $B_{R}$. To build these perturbations, we consider the two following radial compactly supported functions:

$$
\varphi_{R}(x)=\varphi(|x| / R)= \begin{cases}1 & |x| / R<1 / 2 \\ 2-2|x| / R & 1 / 2 \leqslant|x| / R<1 \\ 0 & |x| / R \geqslant 1 .\end{cases}
$$

and

$$
\tilde{\varphi}_{R}(x)=\tilde{\varphi}_{R}(|x|)= \begin{cases}1 & |x|<\sqrt{R} \\ 2-2 \frac{\log (|x|)}{\log R} & \sqrt{R} \leqslant|x|<R \\ 0 & |x| \geqslant R .\end{cases}
$$


For $\boldsymbol{v} \in S^{n-1}$ and $t \in[-1,1]$ we define

$$
\Psi_{R, t}(x):=x+t \varphi_{R}(x) \boldsymbol{v} .
$$

We set

$$
E_{R, t}=\Psi_{R, t}(E) .
$$

Throughout the paper, we denote

$$
u=\chi_{E} \quad \text { and } \quad u_{R, t}(x)=\chi_{E_{R, t}}=u\left(\Psi_{R, t}^{-1}(x)\right) .
$$

Note that these definitions depend upon a fixed unit vector $\boldsymbol{v}$.

Likewise we define $\tilde{\Psi}_{R, t}, \tilde{E}_{R, t}, \tilde{u}_{R, t}$, with $\tilde{\varphi}_{R}$ replacing $\varphi_{R}$.

We prove now the following lemma, which is the appropriate analogue for the nonlocal functional $P_{K, B_{R}}$ of Lemma 1 in 37.

Lemma 2.1. Let $n \geqslant 2, R \geqslant 4$, and $K$ a kernel satisfying (1.3)-(1.7). For every measurable $E \subset \mathbb{R}^{n}$ with $P_{K, B_{R}}(E)<\infty$ we have:

(a) For all $t \in(-1,1)$

$$
P_{K, B_{R}}\left(E_{R, t}\right)+P_{K, B_{R}}\left(E_{R,-t}\right)-2 P_{K, B_{R}}(E) \leqslant 32 \frac{t^{2}}{R^{2}} P_{K^{*}, B_{R}}(E),
$$

where $K^{*}$ is the kernel appearing in (1.7).

(b) For all $t \in(-1,1)$

$$
P_{K, B_{R}}\left(\tilde{E}_{R, t}\right)+P_{K, B_{R}}\left(\tilde{E}_{R,-t}\right)-2 P_{K, B_{R}}(E) \leqslant \frac{(32 \pi t)^{2}}{\log R} \sup _{\rho \in[1, R]} \frac{P_{K^{*}, B_{\rho}}(E)}{\rho^{2}},
$$

where $K^{*}$ is as above.

Proof. We set $A_{R}:=\mathbb{R}^{2 n} \backslash\left(\mathcal{C} B_{R} \times \mathcal{C} B_{R}\right)$.

Let us prove first point (a). We have

$$
P_{K, B_{R}}\left(E_{R, \pm t}\right)=\frac{1}{2} \iint_{A_{R}}\left|u\left(\Psi_{R, \pm t}^{-1}(x)\right)-u\left(\Psi_{R, \pm t}^{-1}(\bar{x})\right)\right|^{2} K(x-\bar{x}) d x d \bar{x} .
$$

Changing variables $y=\Psi_{R, \pm t}^{-1}(x), \bar{y}=\Psi_{R, \pm t}^{-1}(\bar{x})$ in the integral we obtain

$$
P_{K, B_{R}}\left(E_{R, \pm t}\right)=\frac{1}{2} \iint_{A_{R}}|u(y)-u(\bar{y})|^{2} K\left(\Psi_{R, \pm t}(y)-\Psi_{R, \pm t}(\bar{y})\right) J_{ \pm t}(y) d y J_{ \pm t}(\bar{y}) d \bar{y}
$$

where $J_{ \pm t}$ are the Jacobians which, as proven in Lemma 1 in [37], are

$$
J_{ \pm t}(y)=\operatorname{det}\left(D \Psi_{R, \pm t}(y)\right)=1 \pm t \partial_{v} \varphi_{R}(y) .
$$

We call

$$
\varepsilon=\varepsilon(y, \bar{y}, R):=\frac{\varphi(y / R)-\varphi(\bar{y} / R)}{|y-\bar{y}|} .
$$

Note that, since $\|\varphi\|_{C^{0,1}\left(\mathbb{R}^{n}\right)}=2$, we have

$$
|\varepsilon| \leqslant 2 / R \text { and }\left|\partial_{\boldsymbol{v}} \varphi_{R}\right| \leqslant 2 / R .
$$


Let

$$
z=y-\bar{y}
$$

By taking $R \geqslant 4$ we may assume $|\varepsilon| \in(0,1 / 2]$. Then, by the assumption (1.7) on the second derivatives of the kernel we have

$$
K(z \pm t \varepsilon|z| \boldsymbol{v})=K(z) \pm t \partial_{\boldsymbol{v}} K(z) \varepsilon|z|+\boldsymbol{e}_{ \pm}(y, \bar{y}, r),
$$

where

$$
\left|\boldsymbol{e}_{ \pm}\right| \leqslant \frac{1}{2} t^{2} K^{*}(z) \varepsilon^{2}
$$

Therefore,

$$
\begin{aligned}
& K(\left.\Psi_{R, t}(y)-\Psi_{R, t}(\bar{y})\right) J_{t}(y) J_{t}(\bar{y})+K\left(\Psi_{R,-t}(y)-\Psi_{R,-t}(\bar{y})\right) J_{-t}(y) J_{-t}(\bar{y})= \\
&=\left(K(z)+t \partial_{\boldsymbol{v}} K(z) \varepsilon|z|+\boldsymbol{e}_{+}\right)\left(1+t \partial_{\boldsymbol{v}} \varphi_{R}(y)\right)\left(1+t \partial_{\boldsymbol{v}} \varphi_{R}(\bar{y})\right) \\
& \quad+\left(K(z)-t \partial_{\boldsymbol{v}} K(z) \varepsilon|z|+\boldsymbol{e}_{-}\right)\left(1-t \partial_{\boldsymbol{v}} \varphi_{R}(y)\right)\left(1-t \partial_{\boldsymbol{v}} \varphi_{R}(\bar{y})\right) \\
&=2 K(z)+\boldsymbol{e}(y, \bar{y}, r)
\end{aligned}
$$

where

$$
\begin{aligned}
|\boldsymbol{e}|= & \left|2 t^{2} \partial_{\boldsymbol{v}} K(z) \varepsilon\right| z \mid\left(\partial_{\boldsymbol{v}} \varphi_{R}(y)+\partial_{\boldsymbol{v}} \varphi_{R}(\bar{y})\right)+\boldsymbol{e}_{+}+\boldsymbol{e}_{-}+ \\
& +t\left(\boldsymbol{e}_{+}-\boldsymbol{e}_{-}\right)\left(\partial_{\boldsymbol{v}} \varphi_{R}(y)+\partial_{\boldsymbol{v}} \varphi_{R}(\bar{y})\right) \mid+t^{2} \partial_{\boldsymbol{v}} \varphi_{R}(y) \partial_{\boldsymbol{v}} \varphi_{R}(\bar{y})\left[2 K(z)+\boldsymbol{e}_{+}+\boldsymbol{e}_{-}\right] \\
\leqslant & t^{2}\left(2 K^{*}(z)|\varepsilon| \frac{4}{R}+K^{*}(z) \varepsilon^{2}+K^{*}(z) \varepsilon^{2} \frac{4}{R}+\frac{4}{R^{2}} K^{*}(z)\left(2+t^{2} \varepsilon^{2}\right)\right) \\
\leqslant & t^{2} K^{*}(z)\left(\frac{16}{R^{2}}+\frac{4}{R^{2}}+\frac{16}{R^{3}}+\frac{3}{R^{2}}\right) \\
\leqslant & \frac{32 t^{2}}{R^{2}} K^{*}(z) .
\end{aligned}
$$

Here we have used again the assumption (1.7) to estimate terms involving first order derivatives of $K$. In addition, we have used the estimate (2.10) for $e_{ \pm}$, and that $R^{-3} \leqslant R^{-2} / 4$ since $R \geqslant 4$.

Thus, using (2.8), (2.11) and (2.12), we have

$$
\begin{aligned}
P_{K, B_{R}}\left(E_{R, t}\right) & +P_{K, B_{R}}\left(E_{R-t}\right)-2 P_{K, B_{R}}(E) \leqslant \\
\leqslant & \frac{16 t^{2}}{R^{2}} \iint_{A_{R}}|u(y)-u(\bar{y})|^{2} K^{*}(y-\bar{y}) d y d \bar{y}=\frac{32 t^{2}}{R^{2}} P_{K^{*}, B_{R}}(E) .
\end{aligned}
$$

This finishes the proof of (a).

The proof of (b) - i.e. of (2.7) - is almost identical with the the difference that we use the function $\tilde{\varphi}_{R}$ instead of $\varphi_{R}$. More precisely, we consider $\tilde{\Psi}_{R, \pm} t, \tilde{u}_{R, \pm t}$, $\tilde{E}_{R \pm t}$ instead of $\Psi_{R, \pm t}, u_{R, \pm t}, E_{R, \pm t}$. The only important difference is that now (2.9) does not hold since

$$
\left|\nabla \tilde{\varphi}_{R}(x)\right|=\frac{2 \chi_{\{\sqrt{R} \leqslant|x| \leqslant R\}}}{\log R|x|} .
$$


Instead we use

$$
\varepsilon(y, \bar{y}, R) \leqslant \pi \frac{2}{\log R \max \{\sqrt{R}, \rho\}} \quad \text { whenever }(y, \bar{y}) \in \mathbb{R}^{2 n} \backslash A_{\rho}, 1 \leqslant \rho \leqslant R
$$

Note that $\mathbb{R}^{2 n} \backslash A_{\rho}=\{(y, \bar{y}): y \geqslant \rho$ and $\bar{y} \geqslant \rho\}$. The factor $\pi$ appears because we need to apply the mean value theorem connecting $y$ and $\bar{y}$ by a circular arc contained in $\mathbb{R}^{n} \backslash B_{\rho}$.

Similarly,

$$
\max \left\{\left|\partial_{\boldsymbol{v}} \varphi_{R}(y)\right|,\left|\partial_{\boldsymbol{v}} \varphi_{R}(\bar{y})\right|\right\} \leqslant \frac{2}{\log R \max \{\sqrt{R}, \rho\}} \quad \text { for }(y, \bar{y}) \in \mathbb{R}^{2 n} \backslash A_{\rho}, \rho \geqslant 1
$$

Hence, in place of 2.12 we obtain

$$
|\boldsymbol{e}(y, \bar{y}, R)| \leqslant \frac{32 \pi^{2} t^{2}}{(\log R)^{2} \max \left\{R^{2}, \rho^{2}\right\}} K^{*}(z) \quad \text { for }(y, \bar{y}) \in \mathbb{R}^{2 n} \backslash A_{\rho}, \rho \geqslant 1 .
$$

Now, we decompose the domain $A_{R}$ in 2.8 as

$$
A_{R}=A_{\sqrt{R}} \cup \bigcup_{i=k+1}^{2 k} \tilde{A}_{i}
$$

where

$$
k \in \mathbb{N}, \quad \log _{2} R \leqslant 2 k<\log _{2} R+2, \quad \theta^{2 k}=R, \quad \text { and } \quad \tilde{A}_{i}=A_{\theta^{i}} \backslash A_{\theta^{i-1}} .
$$

Note that $\theta \in(1,2]$. Using (2.13) and (2.8) with the previous domain decomposition we obtain

$$
\begin{aligned}
P_{K, B_{R}}\left(\tilde{E}_{R, t}\right) & +P_{K, B_{R}}\left(\tilde{E}_{R,-t}\right)-2 P_{K, B_{R}}(E) \leqslant \\
\leqslant & \frac{32 \pi^{2} t^{2}}{(\log R)^{2}}\left(\frac{1}{R} \iint_{A_{\sqrt{R}}}|u(y)-u(\bar{y})|^{2} K^{*}(y-\bar{y}) d y d \bar{y}\right. \\
& \left.+\sum_{i=k+1}^{2 k} \frac{1}{\theta^{2(i-1)}} \iint_{\tilde{A}_{i}}|u(y)-u(\bar{y})|^{2} K^{*}(y-\bar{y}) d y d \bar{y}\right) \\
\leqslant & \frac{32 \pi^{2} t^{2}}{(\log R)^{2}}\left(\frac{1}{R} P_{K^{*}, B_{\sqrt{R}}}(E)+\sum_{i=k+1}^{2 k} \frac{1}{\theta^{2(i-1)}} P_{K^{*}, B_{\theta^{i}}}(E)\right) .
\end{aligned}
$$


Thus denoting $S:=\sup _{\rho \in[1, R]} \frac{P_{K^{*}, B_{\rho}}(E)}{\rho^{2}}$

$$
\begin{aligned}
P_{K, B_{R}}\left(\tilde{E}_{R}^{t}\right)+P_{K, B_{R}}\left(\tilde{E}_{R}^{-t}\right)-2 P_{K, B_{R}}(E) & \leqslant \frac{32 \pi^{2} t^{2}}{(\log R)^{2}}\left(S+\sum_{i=k+1}^{2 k} \frac{\theta^{2 i}}{\theta^{2(i-1)}} S\right) \\
& \leqslant \frac{32 \pi^{2} t^{2}}{(\log R)^{2}} \theta^{2}(k+1) S \\
& \leqslant \frac{32 \pi^{2} t^{2}}{(\log R)^{2}} 4\left(\log _{2}(4 R)+1\right) S \\
& \leqslant \frac{(32 \pi t)^{2}}{\log R} S
\end{aligned}
$$

This finishes the proof of 2.7 - and thus of (b).

The following lemma is a key step in the proof of our main results: given a minimizer $E$ and any possible competitor $F$, it allows to "measure" the interaction between points in $E \backslash F$ and points in $F \backslash E$ in terms of the difference between the $K$-perimeter of $F$ and the $K$-perimeter of $E$. Here we see that the nonlocality of the functional plays a crucial role.

Lemma 2.2. Let $E, F \subset \mathbb{R}^{n}$. Assume that $E$ is a minimizer for $P_{K, B_{R}}$ and that $F$ coincides with $E$ outside of $B_{R}$, that is, $E \backslash B_{R}=F \backslash B_{R}$. Assume moreover that

$$
P_{K, B_{R}}(F) \leqslant P_{K, B_{R}}(E)+\delta,
$$

for some $\delta \geqslant 0$.

Then,

$$
2 L_{K}(F \backslash E, E \backslash F) \leqslant \delta
$$

Proof. Let $C=E \cup F$ and $D=E \cap F$. Note that both $C$ and $D$ coincides with $E$ and $F$ outside of $B_{R}$. By a direct computation we find that

$$
P_{K, B_{R}}(C)+P_{K, B_{R}}(D)+2 L_{K}(F \backslash E, E \backslash F)=P_{K, B_{R}}(E)+P_{K, B_{R}}(F) .
$$

Using (2.14) and the minimality of $E$, we deduce

$$
P_{K, B_{R}}(E)+P_{K, B_{R}}(F) \leqslant 2 P_{K, B_{R}}(E)+\delta \leqslant P_{K, B_{R}}(C)+P_{K, B_{R}}(D)+\delta,
$$

which, together with 2.15), concludes the proof of the Lemma.

It is worth to observe that, in spite of its simplicity, the identity in 2.15 has consequences that seem to be interesting in themselves, such as the fact that minimizers are included one in the other, as stated in the following result:

Lemma 2.3 (Mutual inclusion of minimizers). Assume that $E$ and $F$ are minimizers for $P_{K, \Omega}$, with $E \backslash \Omega=F \backslash \Omega$. Suppose that $K(y)>0$ for $|y|<\operatorname{diam}(\Omega)$. Then, either $E \subseteq F$ or $F \subseteq E$. 
Proof. The minimality of the sets give that

$$
P_{K, \Omega}(E) \leqslant P_{K, \Omega}(E \cup F) \text { and } P_{K, \Omega}(F) \leqslant P_{K, \Omega}(E \cap F) .
$$

Then, using (2.15),

$$
2 L_{K}(F \backslash E, E \backslash F)=P_{K, \Omega}(E)+P_{K, \Omega}(F)-P_{K, \Omega}(E \cup F)-P_{K, \Omega}(E \cap F) \leqslant 0,
$$

which implies that one between $F \backslash E$ and $E \backslash F$ has necessarily zero measure.

The following lemma is the analogue of Lemma 2.2 but under the assumption that $E$ is a stable set (not necessarily a minimizer) for the $K$-perimeter.

Lemma 2.4. Let $E \subset \mathbb{R}^{n}$. Assume that $E$ is a stable set for $P_{K, B_{R}}$ and that $F_{t}=\Psi_{t}(E)$, where $\Psi_{t}$ is the integral flow of some vector field $X \in C_{c}^{2}\left(B_{R} ; \mathbb{R}^{n}\right)$. Assume moreover that

$$
P_{K, B_{R}}\left(F_{t}\right)+P_{K, B_{R}}\left(F_{-t}\right) \leqslant 2 P_{K, B_{R}}(E)+\eta t^{2}, \quad \text { for } t \in(-1,1)
$$

for some $\eta>0$.

Then, for any $\varepsilon>0$ there exists $t_{0}>0$ such that for $t \in\left(-t_{0}, t_{0}\right)$

$$
\min \left\{L_{K}\left(F_{t} \backslash E, E \backslash F_{t}\right), L_{K}\left(F_{-t} \backslash E, E \backslash F_{-t}\right)\right\} \leqslant(\eta / 4+\varepsilon) t^{2} .
$$

Proof. Let $C_{t}=E \cup F_{t}$ and $D_{t}=E \cap F_{t}$. Note that both $C_{t}$ and $D_{t}$ coincides with $E$ and $F_{t}$ outside of $B_{R}$. We have

$$
P_{K, B_{R}}\left(C_{t}\right)+P_{K, B_{R}}\left(D_{t}\right)+2 L_{K}\left(F_{t} \backslash E, E \backslash F_{t}\right)=P_{K, B_{R}}(E)+P_{K, B_{R}}\left(F_{t}\right) .
$$

and

$$
P_{K, B_{R}}\left(C_{-t}\right)+P_{K, B_{R}}\left(D_{-t}\right)+2 L_{K}\left(F_{-t} \backslash E, E \backslash F_{-t}\right)=P_{K, B_{R}}(E)+P_{K, B_{R}}\left(F_{-t}\right) .
$$

Using (2.16) and the stability of $E$, we deduce

$$
\begin{aligned}
P_{K, B_{R}}\left(C_{t}\right)+ & P_{K, B_{R}}\left(D_{t}\right)+P_{K, B_{R}}\left(C_{-t}\right)+P_{K, B_{R}}\left(D_{-t}\right) \\
& +2 L_{K}\left(F_{t} \backslash E, E \backslash F_{t}\right)+2 L_{K}\left(F_{-t} \backslash E, E \backslash F_{-t}\right) \leqslant \\
\leqslant & 4 P_{K, B_{R}}(E)+\eta t^{2} \\
\leqslant & P_{K, B_{R}}\left(C_{t}\right)+P_{K, B_{R}}\left(D_{t}\right)+P_{K, B_{R}}\left(C_{-t}\right)+P_{K, B_{R}}\left(D_{-t}\right)+(\eta+4 \varepsilon) t^{2}
\end{aligned}
$$

for $t \in\left(-t_{0}, t_{0}\right)$ with $t_{0}>0$ small enough (depending on $E$ and $X$ ).

We remind that the definition of $E_{R, t}$ depends on the choice of the vector $\boldsymbol{v} \in S^{n-1}$ along which we are translating the set $E$ - see (2.3) and (2.4). In the sequel, we will use the notion of directional derivative of a $\overline{B V}$-function in the distributional sense. Let $u \in B V(\Omega)$ and $\boldsymbol{v} \in S^{n-1}$; we define:

$$
\left|\partial_{\boldsymbol{v}} u\right|(\Omega):=\sup \left\{-\int_{\Omega} u(x) \partial_{\boldsymbol{v}} \phi(x) d x: \phi \in C_{c}^{1}(\Omega,[-1,1])\right\},
$$

and

$$
\left(\partial_{\boldsymbol{v}} u\right)_{ \pm}(\Omega):=\sup \left\{\mp \int_{\Omega} u(x) \partial_{\boldsymbol{v}} \phi(x) \boldsymbol{d} x: \phi \in C_{c}^{1}(\Omega,[0,1])\right\} .
$$


The following lemma will allow us to obtain geometric informations from the conclusion of Lemma 2.4.

Lemma 2.5. Let $n \geqslant 2, \eta>0, E \subset \mathbb{R}^{n}$ measurable. Assume that for all $\boldsymbol{v} \in S^{n-1}$, there exists a sequence $t_{k} \rightarrow 0, t_{k} \in(-1,1)$ such that

$$
\lim _{k \rightarrow \infty} \frac{1}{t_{k}^{2}}\left|\left\{\left(E+t_{k} \boldsymbol{v}\right) \backslash E\right\} \cap B_{1}\right| \cdot\left|\left\{E \backslash\left(E+t_{k} \boldsymbol{v}\right)\right\} \cap B_{1}\right| \leqslant \frac{\eta}{4}
$$

Then,

(a) The characteristic function $u=\chi_{E}$ has finite total variation in $B_{1}$, that is, $u \in \operatorname{BV}\left(B_{1}\right)$.

(b) For all $\boldsymbol{v} \in S^{n-1}$, the distributional derivative $\partial_{\boldsymbol{v}} u$ is a signed measure on $B_{1}$ of the form

$$
\partial_{\boldsymbol{v}} u=\left(\partial_{\boldsymbol{v}} u\right)_{+}-\left(\partial_{\boldsymbol{v}} u\right)_{-}
$$

with

$$
\left(\partial_{\boldsymbol{v}} u\right)_{ \pm}:=\left.\left(-\nu_{E} \cdot \boldsymbol{v}\right)_{ \pm} H^{n-1}\right|_{\partial^{*} E \cap B_{1}}
$$

where $\partial^{*} E$ is the reduced boundary of $E$.

(c) For all $\boldsymbol{v} \in S^{n-1}$

$$
\min \left\{\int_{B_{1}}\left(\partial_{v} u\right)_{+} d x, \int_{B_{1}}\left(\partial_{v} u\right)_{-} d x\right\} \leqslant \frac{\sqrt{\eta}}{2}
$$

and

$$
\max \left\{\int_{B_{1}}\left(\partial_{\boldsymbol{v}} u\right)_{+} d x, \int_{B_{1}}\left(\partial_{\boldsymbol{v}} u\right)_{-} d x\right\} \leqslant 2\left|B_{1}^{(n-1)}\right|+\frac{\sqrt{\eta}}{2},
$$

where $\left|B_{1}^{(n-1)}\right|$ denotes the $n-1$-dimensional volume of the ball $B_{1} \subset \mathbb{R}^{n-1}$.

(d) $\operatorname{Per}_{B_{1}}(E)=H^{n-1}\left(\partial^{*} E \cap B_{1}\right) \leqslant\left|S^{n-1}\right|\left(1+\frac{\sqrt{\eta}}{2\left|B_{1}^{(n-1)}\right|}\right)$.

We next give the

Proof of Lemma 2.5. We have

$$
\frac{1}{\left|t_{k}\right|} \min \left\{\left|\left\{\left(E+t_{k} \boldsymbol{v}\right) \backslash E\right\} \cap B_{1}\right|,\left|\left\{E \backslash\left(E+t_{k} \boldsymbol{v}\right)\right\} \cap B_{1}\right|\right\} \leqslant \frac{\sqrt{\eta}}{2} .
$$

Denoting $u=\chi_{E}, 2.20$ becomes

$$
\frac{1}{\left|t_{k}\right|} \min \left\{\int_{B_{1}}\left(u\left(x-t_{k} \boldsymbol{v}\right)-u(x)\right)_{+} d x, \int_{B_{1}}\left(u\left(x-t_{k} \boldsymbol{v}\right)-u(x)\right)_{-} d x\right\} \leqslant \frac{\sqrt{\eta}}{2}
$$

Let us now denote the measures

$$
\mu_{k, \pm}(d x)=\left(\frac{u\left(x-t_{k} \boldsymbol{v}\right)-u(x)}{-t_{k}}\right)_{ \pm} d x
$$


and $\mu_{k}=\mu_{k,+}-\mu_{k,-}$. Note that

$$
\mu_{k}\left(B_{1}\right)=\int_{B_{1}} \frac{u\left(x-t_{k} \boldsymbol{v}\right)-u(x)}{-t_{k}} d x=\frac{\int_{B_{1}+t_{k} \boldsymbol{v}} u d x-\int_{B_{1}} u d x}{t_{k}} .
$$

Hence, since $u$ is a characteristic function,

$$
\left|\mu_{k}\left(B_{1}\right)\right| \leqslant \frac{2\left|\left(B_{1}+t_{k} \boldsymbol{v}\right) \backslash B_{1}\right|}{\left|t_{k}\right|} \leqslant 2\left|B_{1}^{(n-1)}\right|,
$$

where $\left|B_{1}^{(n-1)}\right|$ denotes the $(n-1)$-dimensional volume of the ball $B_{1}^{(n-1)} \subset \mathbb{R}^{n-1}$.

Now, by 2.21) we have

$$
\min \left\{\mu_{k,+}\left(B_{1}\right), \mu_{k,-}\left(B_{1}\right)\right\} \leqslant \frac{\sqrt{\eta}}{2} .
$$

But then, since $\mu_{k}=\mu_{k,+}-\mu_{k,-}$ we must have

$$
\max \left\{\mu_{k,+}\left(B_{1}\right), \mu_{k,-}\left(B_{1}\right)\right\} \leqslant 2\left|B_{1}^{(n-1)}\right|+\frac{\sqrt{\eta}}{2} .
$$

This implies that both the (nonegative) measures $\mu_{k,+}, \mu_{k,-}$ are bounded in $B_{1}$ independently of $k$. Thus, up to extracting a subsequence, we have $\mu_{k,+} \rightarrow \mu_{+}$and $\mu_{k,-} \rightarrow \mu_{-}$(weak convergence) for some bounded nonnegative measures $\mu_{+}, \mu_{-}$.

We have clearly that $\mu_{k} \rightarrow \mu_{+}-\mu_{-}$. Moreover it is immediate to check that, for every $\eta \in C_{c}^{\infty}\left(B_{1}\right)$

$$
\int_{B_{1}} \eta(x) \mu_{k}(d x)=\int_{B_{1}} \frac{\eta\left(x+t_{k} \boldsymbol{v}\right)-\eta(x)}{-t_{k}} u(x) d x
$$

if $t_{k}$ is smaller than dist $\left(\operatorname{spt} \eta, \partial B_{1}\right)$, where $\operatorname{spt} \eta$ denotes the support of $\eta$ and $\operatorname{dist}(A, B)$ the distance between the sets $A$ and $B$. It follows that

$$
\lim _{k \rightarrow \infty} \int_{B_{1}} \eta(x) \mu_{k}(d x)=-\int_{B_{1}} \partial_{\boldsymbol{v}} \eta(x) u(x) d x
$$

and thus $\mu_{+}-\mu_{-}$is the distributional derivative of $u$ in the direction $\boldsymbol{v}$ restricted to $B_{1}$, which we denote $\partial_{\boldsymbol{v}} u$.

Moreover from (2.22) and (2.23) it follows that

$$
\min \left\{\mu_{+}\left(B_{1}\right), \mu_{-}\left(B_{1}\right)\right\} \leqslant \frac{\sqrt{\eta}}{2}
$$

and

$$
\max \left\{\mu_{+}\left(B_{1}\right), \mu_{-}\left(B_{1}\right)\right\} \leqslant 2\left|B_{1}^{(n-1)}\right|+\frac{\sqrt{\eta}}{2} .
$$

The above inequalities hold for translations in any direction $\boldsymbol{v} \in S^{n-1}$, and hence we can choose $\boldsymbol{v}$ to be the coordinate unit vectors. We then obtain that there are $n$ signed measures $\boldsymbol{\mu}=\left(\mu_{1}, \mu_{2}, \ldots, \mu_{n}\right)$ in $B_{1}$ such that

$$
\left|\mu_{i}\right|\left(B_{1}\right) \leqslant 2\left|B_{1}^{(n-1)}\right|+\sqrt{2 \eta}, \quad \text { for } i=1, \ldots, n
$$


Moreover, since by definition $\mu_{i}$ is the distributional derivative $\partial_{i} u$ we have

$$
\sum_{i} \int_{B_{1}} T_{i} \mu_{i}(d x)=-\int_{B_{1}}(\operatorname{div} \boldsymbol{T}) u d x
$$

for every vector field $\boldsymbol{T} \in C_{c}^{1}\left(B_{1} ; \mathbb{R}^{n}\right)$, where $u=\chi_{E}$. This proves (a). Namely, $u \in \mathrm{BV}\left(B_{1}\right)$.

We next prove (b). Using that $\partial_{\boldsymbol{v}} u$ is the distributional derivative of $u=\chi_{E}$ and applying the divergence theorem for sets of finite perimeter — see [23] — we have, for all $\varphi \in C_{c}^{1}\left(B_{1}\right)$,

$$
\begin{aligned}
\int_{B_{1}} \varphi \partial_{\boldsymbol{v}} u d x & =-\int_{B_{1}} \partial_{\boldsymbol{v}} \varphi u d x \\
& =-\int_{B_{1} \cap E} \operatorname{div}(\varphi \boldsymbol{v}) \varphi \\
& =-\int_{\partial^{*} E} \varphi\left(\nu_{E} \cdot \boldsymbol{v}\right) d H^{n-1},
\end{aligned}
$$

where $\partial^{*} E$ denotes the reduced boundary of $E$ (in $B_{1}$ ).

The identity 2.25) gives the decomposition $\partial_{\boldsymbol{v}} u=\left(\partial_{\boldsymbol{v}} u\right)_{+}-\left(\partial_{\boldsymbol{v}} u\right)_{-}$. for

$$
\left(\partial_{\boldsymbol{v}} u\right)_{ \pm}=-\left.\left(\nu_{E} \cdot \boldsymbol{v}\right)_{ \pm} H^{n-1}\right|_{\partial^{*} E \cap B_{1}} .
$$

Note that the previous decomposition is the Hahn-Jordan decomposition of $\partial_{\boldsymbol{v}} u$ since $\left(\partial_{\boldsymbol{v}} u\right)_{+}$and $\left(\partial_{\boldsymbol{v}} u\right)_{-}$are concentrated on disjoint subsets of $\partial^{*} E$. In particular we deduce that $\left(\partial_{v} u\right)_{ \pm} \leqslant \mu_{ \pm}$. Thus (b) and (c) follow. Namely, with the above definitions we have

$$
\min \left\{\int_{B_{1}}\left(\partial_{\boldsymbol{v}} u\right)_{+} d x, \int_{B_{1}}\left(\partial_{\boldsymbol{v}} u\right)_{-} d x\right\} \leqslant \frac{\sqrt{\eta}}{2}
$$

and

$$
\max \left\{\int_{B_{1}}\left(\partial_{\boldsymbol{v}} u\right)_{+} d x, \int_{B_{1}}\left(\partial_{\boldsymbol{v}} u\right)_{-} d x\right\} \leqslant 2\left|B_{1}^{(n-1)}\right|+\frac{\sqrt{\eta}}{2},
$$

where $\left|B_{1}^{(n-1)}\right|$ denotes the $n-1$-dimensional volume of the ball $B_{1} \subset \mathbb{R}^{n-1}$.

To prove (d) we integrate with respect to all directions $\boldsymbol{v} \in S^{n-1}$ the inequality

$$
\int_{\partial^{*} E}\left|\nu_{E}(x) \cdot \boldsymbol{v}\right| d H^{n-1}(x) \leqslant 2\left|B_{1}^{(n-1)}\right|+\sqrt{\eta},
$$

which follows from the previous steps. Using Fubini we find

$$
\begin{aligned}
H^{n-1}\left(\partial^{*} E\right) 2\left|B_{1}^{(n-1)}\right| & =\int_{\partial^{*} E} d H^{n-1}(x) \int_{S^{n-1}} d H^{n-1}(\boldsymbol{v})\left|\nu_{E} \cdot \boldsymbol{v}\right| \\
& =\int_{S^{n-1}} d H^{n-1}(\boldsymbol{v}) \int_{\partial^{*} E} d H^{n-1}(x)\left|\nu_{E}(x) \cdot \boldsymbol{v}\right| \\
& \leqslant\left|S^{n-1}\right|\left(2\left|B_{1}^{(n-1)}\right|+\sqrt{\eta}\right),
\end{aligned}
$$


concluding the proof of $(d)$.

\section{Proof of Theorems 1.7 and 1.9}

In this section we give the proof of our uniform $B V$-estimates.

We start with the proof of our general result Theorem 1.9 .

Proof of Theorem 1.9. For the proof we just need to combine Lemma 2.1 (a), Lemma 2.4. and Lemma 2.5. More precisely, by Lemma 2.1 (a) (applied with $R=4$ ), we have that

$$
P_{K, B_{4}}\left(E_{4, t}\right)+P_{K, B_{4}}\left(E_{4,-t}\right)-2 P_{K, B_{4}}(E) \leqslant 2 t^{2} P_{K^{*}, B_{4}}(E) .
$$

Hence, $E$ satisfies the assumption in Lemma 2.4 and therefore, for any $\varepsilon>0$ there exists $t_{0}$ such that for any $t \in\left(0, t_{0}\right)$

$$
\min \left\{L_{K}\left(F_{t} \backslash E, E \backslash F_{t}\right), L_{K}\left(F_{-t} \backslash E, E \backslash F_{-t}\right)\right\} \leqslant(\eta / 4+\varepsilon) t^{2},
$$

with

$$
\eta=2 P_{K^{*}, B_{4}}(E)
$$

Now using the assumption (1.6), namely that $K \geqslant 1$ in $B_{2}$ and the definition of $L_{K}$ we prove that there is a some sequece $t_{k} \in(-1,1)$ with $t_{k} \downarrow 0$ such that

$$
\lim _{k \rightarrow \infty} \frac{1}{t_{k}^{2}}\left|\left\{\left(E+t_{k} \boldsymbol{v}\right) \backslash E\right\} \cap B_{1}\right| \cdot\left|\left\{E \backslash\left(E+t_{k} \boldsymbol{v}\right)\right\} \cap B_{1}\right| \leqslant \frac{\eta}{4}+\varepsilon
$$

for all $\varepsilon>0$.

After letting $\varepsilon \rightarrow 0$, we apply Lemma 2.5 and, in particular, from point (d) we deduce that

$$
\operatorname{Per}_{B_{1}}(E) \leqslant\left|S^{n-1}\right|\left(1+\frac{\sqrt{2 P_{K^{*}, B_{4}}(E)}}{2\left|B_{1}^{n-1}\right|}\right) \leqslant \sqrt{2} n \sqrt{P_{K^{*}, B_{4}}(E)}+\left|S^{n-1}\right|,
$$

as wanted.

In the proof of Theorem 1.7 we will need the following abstract Lemma. Although this useful abstract statement is due of L. Simon [41], the result was previously wellknown in concrete situations, such as in the context of adimensional Hölder norms and their interpolation inequalities. We include its proof here for completeness.

Lemma 3.1. Let $\beta \in \mathbb{R}$ and $C_{0}>0$. Let $S: \mathcal{B} \rightarrow[0,+\infty]$, be a nonnegative function defined on the class $\mathcal{B}$ of open balls $B \subset \mathbb{R}^{n}$ and satisfying the following subadditivity property

$$
B \subset \bigcup_{j=1}^{N} B_{j} \quad \Longrightarrow \quad S(B) \leqslant \sum_{j=1}^{N} S\left(B_{j}\right)
$$

Assume that

$$
S\left(B_{1}\right)<\infty
$$


There is $\delta=\delta(n, \beta)$ such that if

$$
\rho^{\beta} S\left(B_{\rho / 4(z)}\right) \leqslant \delta \rho^{\beta} S\left(B_{\rho}(z)\right)+C_{0} \quad \text { whenever } B_{\rho}(z) \subset B_{1}
$$

Then

$$
S\left(B_{1 / 2}\right) \leqslant C C_{0}
$$

where $C=C(n, \beta)$.

Proof. Define

$$
Q:=\sup _{B_{\rho}(z) \subset B_{3 / 4}} \rho^{\beta} S\left(B_{\rho} / 4(z)\right)
$$

We prove first that $Q<\infty$ since $S\left(B_{1}\right)<\infty$. Take $z \in B_{3 / 4}$. By subadditivity $S\left(B_{1 / 4}(z)\right) \leqslant S\left(B_{1}\right)<\infty$. We define

$$
S^{\prime}(B)=\left(\frac{\operatorname{diam}(\mathrm{B})}{2}\right)^{\beta} S(B) .
$$

Clearly, $S^{\prime}\left(B_{1 / 4}(z)\right)=(1 / 4)^{\beta} S\left(B_{1 / 4}(z)\right) \leqslant 4^{-\beta} S\left(B_{1}\right)$.

On the other hand, by $(3.3)$ we have

$$
S^{\prime}\left(B_{2^{-k-2}}(z)\right) \leqslant \delta S^{\prime}\left(B_{2^{-k}}(z)\right)+C_{0}
$$

and thus, if $\delta \leqslant 1 / 2$, iterating we obtain

$$
S^{\prime}\left(B_{2^{-2 k-2}}(z)\right) \leqslant S^{\prime}\left(B_{1 / 4}(z)\right)+C_{0}<\infty,
$$

for all $k \geqslant 0$. But for $r \in\left(2^{-2(k+1)-2}, 2^{-2 k-2}\right)$ we have

$$
\begin{aligned}
S^{\prime}\left(B_{r}(z)\right) & \leqslant \max \left\{1,4^{-\beta}\right\} S^{\prime}\left(B_{2^{-2 k-2}}(z)\right) \\
& \leqslant \max \left\{1,4^{-\beta}\right\}\left(S^{\prime}\left(B_{1 / 4}(z)\right)+C_{0}\right) \\
& \leqslant \max \left\{1,4^{-\beta}\right\}\left(4^{-\beta} S\left(B_{1}\right)+C_{0}\right)
\end{aligned}
$$

Thus,

$$
Q \leqslant \max \left\{1,4^{-\beta}\right\}\left(4^{-\beta} S\left(B_{1}\right)+C_{0}\right)<\infty .
$$

Let us now fix a finite covering of $\overline{B_{1 / 4}}$ by a universal number $M=M(n)$ of balls of radius $1 / 32$ centered at points of $x_{i} \in \bar{B}_{1 / 4}$, that is

$$
\overline{B_{1 / 4}} \subset \bigcup_{i=1}^{M} B_{1 / 32}\left(x_{i}\right)
$$


Now, using the subaditivity of $S$ and assumption 3.3 we have

$$
\begin{aligned}
\rho^{\beta} S\left(B_{\rho / 4}(z)\right) & \leqslant 8^{\beta} \sum_{i=1}^{M}(\rho / 8)^{\beta} S\left(B_{\rho / 32}\left(z+\rho x_{i}\right)\right) \\
& \leqslant 8^{\beta} \sum_{i=1}^{M}\left(\delta(\rho / 8)^{\beta} S\left(B_{\rho / 8}\left(z+\rho x_{i}\right)\right)+C_{0}\right) \\
& =2^{\beta} \delta \sum_{i=1}^{M} \delta(4 \rho / 8)^{\beta} S\left(B_{\rho / 8}\left(z+\rho x_{i}\right)\right)+8^{\beta} M C_{0} \\
& \leqslant 2^{\beta} \delta M Q+8^{\beta} M C_{0},
\end{aligned}
$$

where we have used that if $B_{\rho}(z) \subset B_{3 / 4}$ then also $B_{4 \rho / 8}\left(z+\rho x_{i}\right) \subset B_{3 \rho / 4}(z) \subset B_{3 / 4}$ and the definition of $Q$. Thus, taking supremum for all balls $B_{\rho}(z) \subset B_{3 / 4}$ in the left hand side we obtain

$$
Q \leqslant 2^{\beta} \delta M Q+8^{\beta} M C_{0}
$$

and for $\delta=2^{\beta-1} / M$ we obtain $Q / 2 \leqslant 8^{\beta} M C_{0}$, which clearly implies the desired bound on $S\left(B_{1 / 2}\right)$.

We will also use the following standard fact.

Lemma 3.2. Let $E \subset \mathbb{R}^{n}$ be measurable and $\Omega \subset \mathbb{R}^{n}$ be smooth. Let

$$
\tilde{P}_{s, B}(E):=\int_{E \cap B} \int_{B \backslash E} \frac{d x d \bar{x}}{|x-\bar{x}|^{n+s}} \text {. }
$$

Then,

$$
\tilde{P}_{s, \Omega}(E) \leqslant C \operatorname{Per}_{\Omega}(E) .
$$

Proof. By [21], Proposition 2.2 and applying the Poincaré-Wirtinger inequality we have that

$$
\left\|u-\bar{u}_{\Omega}\right\|_{W^{s, 1}(\Omega)} \leqslant C\left\|u-\bar{u}_{\Omega}\right\|_{W^{1,1}(\Omega)} \leqslant C \int_{\Omega}|\nabla u| d x
$$

where $\bar{u}$ denotes the average of $u$ in $\Omega$.

By the density of $W^{1,1}(\Omega)$ in $B V(\Omega)$ (see Theorem 1.17 in [27]), (3.6) holds with the right-hand side replaced by $|\nabla u|(\Omega)$. Therefore, for $u=\chi_{E}$, we have

$$
\tilde{P}_{s, \Omega}(E)=\frac{1}{2} \int_{\Omega} \int_{\Omega} \frac{|u(x)-u(\bar{x})|}{|x-\bar{x}|^{n+s}} d x d \bar{x} \leqslant\left\|u-\bar{u}_{\Omega}\right\|_{W^{s, 1}(\Omega)} \leqslant C|\nabla u|(\Omega)=C \operatorname{Per}_{\Omega}(E),
$$

as desired.

Proof of Theorem 1.7. Multiplying the kernel $K \in \mathcal{L}_{2}$ by a positive constant, we may assume that $\lambda \geqslant 2^{n+s}$ and hence $K$ satisfies $\left.1.3-1.7\right)$ with $K^{*}=C_{1} K$.

Therefore, by Theorem 1.9 , we immediately deduce that

$$
\operatorname{Per}_{B_{1}}(E) \leqslant C\left(1+\sqrt{P_{K, B_{4}}(E)}\right)<+\infty
$$


where $\operatorname{Per}_{B_{1}}$ denotes the classical perimeter in $B_{1}$ and $C$ depends only on $n, s, \lambda$ and $\Lambda$ - since $C_{1}$ depends only on these constants.

Now, since $K \in \mathcal{L}_{2}$ and by Lemma 3.2, we deduce that

$$
\begin{aligned}
P_{K, B_{4}}(E) & \leqslant \Lambda P_{s, B_{4}}(E) \\
& \leqslant \Lambda \int_{E \cap B_{4}} \int_{B_{4} \backslash E} \frac{d x d \bar{x}}{|x-\bar{x}|^{n+s}}+\Lambda \int_{B_{4}} \int_{\mathbb{R}^{n} \backslash B_{4}} \frac{d x d \bar{x}}{|x-\bar{x}|^{n+s}} \\
& \leqslant \Lambda \tilde{P}_{s, B_{4}}(E)+C \\
& \leqslant C\left(1+\operatorname{Per}_{B_{4}}(E)\right),
\end{aligned}
$$

where $\tilde{P}_{s, B_{4}}(E)$ is defined as in $(3.4)$.

Hence, 3.7), (3.8) and Young's inequality imply that

$$
\begin{aligned}
\operatorname{Per}_{B_{1}}(E) & \leqslant C\left(1+\left(1+\operatorname{Per}_{B_{4}}(E)\right)^{1 / 2}\right) \\
& \leqslant C\left(1+\delta^{-1}\right)+\delta \operatorname{Per}_{B_{4}}(E),
\end{aligned}
$$

for all $\delta>0$, where $C$ depends only on $n, s, \lambda$, and $\Lambda$.

Next, we observe that, since $E$ is a stable minimal set for $P_{K, B_{1}}$, with $K \in$ $\mathcal{L}_{2}(s, \lambda, \Lambda)$, given $B_{r}(z) \subset B_{1}$ then the rescaled set $E^{\prime}=(r / 4)^{-1}(E-z)$ is a stable minimal set for $P_{K^{\prime}, B_{4}}$, where

$$
K^{\prime}(y):=(r / 4)^{n+s} K(r y / 4) \quad \text { belongs again to } \mathcal{L}_{2}(s, \lambda, \Lambda) .
$$

Thus, rescaling the estimates (3.9) applied to $E^{\prime}$ we obtain, for $E$,

$$
r^{1-n} \operatorname{Per}_{B_{r / 4}(z)}(E) \leqslant C(n, s, \lambda, \Lambda, \delta)+\delta r^{1-n} \operatorname{Per}_{B_{r}(z)}(E) .
$$

Therefore, considering the subadditive function on the class of balls

$$
S(B):=\operatorname{Per}_{B}(E)
$$

and taking $\beta:=1-n$, and $\delta=\delta(n, \beta)$ given by Lemma 3.1 we find that

$$
S\left(B_{1 / 2}\right) \leqslant C(n, s, \lambda, \Lambda)
$$

since $S\left(B_{1}\right)<+\infty$ by $(3.7)$ - note since $E$ is a stable minimal set in $B_{4}$ by definition we have $P_{K, B_{4}}(E)<+\infty$.

Thus, we have shown that

$$
\operatorname{Per}_{B_{1 / 4}}(E) \leqslant C(n, s, \lambda, \Lambda)
$$

where $C(n, s, \lambda, \Lambda)$ is a universal constant depending only on $n, s, \lambda, \Lambda$.

By scaling and using a standard covering argument, we obtain

$$
\operatorname{Per}_{B_{1}}(E) \leqslant C(n, s, \lambda, \Lambda),
$$

which finishes the proof. 
Proof of Corollary 1.8. We combine the universal perimeter estimate in $B_{1}$ of Theorem 1.7 - see (3.11) - with the "interpolation inequality" $P_{K, B_{1}}(E) \leqslant C\left(1+\operatorname{Per}_{B_{1}}(E)\right)$, shown in $(3.8)$, to obtain $P_{K, B_{1}}(E) \leqslant C$. The estimate for the $K$-perimeter in $B_{R}$ then follows using the scaling invariance of the class $\mathcal{L}_{2}(s, \lambda, \Lambda)$.

\section{Proof of Theorems 1.14 and 1.19}

Before giving the proofs of Theorems 1.14 and 1.19 , we give some preliminary lemmas. We start with the following easy fact, that we state explicitly since we will use it several times later on.

Remark 4.1. Let $\Phi$ be a continuous and odd function defined on the $m$-dimensional sphere $S^{m}$, with $m \geqslant 1$.

Then, there exists $\boldsymbol{v}^{*} \in S^{m}$ such that $\Phi\left(\boldsymbol{v}^{*}\right)=0$.

The proof of this fact is obvious since $S^{m}$ is connected when $m \geqslant 1$.

Lemma 4.2. Suppose that $\Phi_{+}$and $\Phi_{-}$are two continuous functions defined on $S^{n-1}$, which satisfy

$$
\Phi_{+}(-\boldsymbol{v})=\Phi_{-}(\boldsymbol{v}) \quad \text { for any } \boldsymbol{v} \in S^{n-1} .
$$

Assume moreover that there exists $\mu>0$ such that for any $\boldsymbol{v} \in S^{n-1}$

$$
\min \left\{\Phi_{+}(\boldsymbol{v}), \Phi_{-}(\boldsymbol{v})\right\} \leqslant \mu .
$$

Then, after a rotation of coordinates, we have that

$$
\max \left\{\Phi_{+}\left(\boldsymbol{e}_{i}\right), \Phi_{-}\left(\boldsymbol{e}_{i}\right)\right\} \leqslant \mu \quad \text { for } 1 \leqslant i \leqslant n-1,
$$

where $\boldsymbol{e}_{i}$ denote the standard basis of $\mathbb{R}^{n}$,

Proof. For $\boldsymbol{v} \in S^{n-1}$, we consider the function

$$
\Phi(\boldsymbol{v})=\Phi_{+}(\boldsymbol{v})-\Phi_{-}(\boldsymbol{v}) .
$$

Using (4.1), it is easy to verify that $\Phi$ is odd and hence, using Remark 4.1, there exists a vector $\boldsymbol{v}_{1}^{*} \in S^{n-1}$ for which

$$
\Phi\left(\boldsymbol{v}_{1}^{*}\right)=\Phi_{+}\left(\boldsymbol{v}_{1}^{*}\right)-\Phi_{-}\left(\boldsymbol{v}_{1}^{*}\right)=0 .
$$

This clearly implies that

$$
\Phi_{+}\left(\boldsymbol{v}_{1}^{*}\right)=\Phi_{-}\left(\boldsymbol{v}_{1}^{*}\right)=\min \left\{\Phi_{+}\left(\boldsymbol{v}_{1}^{*}\right), \Phi_{-}\left(\boldsymbol{v}_{1}^{*}\right)\right\}=\max \left\{\Phi_{+}\left(\boldsymbol{v}_{1}^{*}\right), \Phi_{-}\left(\boldsymbol{v}_{1}^{*}\right)\right\} .
$$

Hence, by (4.2), we deduce that

$$
\max \left\{\Phi_{+}\left(\boldsymbol{v}_{1}^{*}\right), \Phi_{-}\left(\boldsymbol{v}_{1}^{*}\right)\right\} \leqslant \mu .
$$

Now we define $\Phi_{2}$ to be $\Phi$ restricted to the $(n-2)$-dimensional sphere given by $S^{n-1} \cap$ $\left(v_{1}^{*}\right)^{\perp}$. By Remark 4.1 applied now to $\Phi_{2}$, there exists a vector $\boldsymbol{v}_{2}^{*} \in S^{n-1} \cap\left(v_{1}^{*}\right)^{\perp}$ for which (4.4) holds (with $\boldsymbol{v}_{1}^{*}$ replaced by $\boldsymbol{v}_{2}^{*}$ ). We can iterate this procedure $(n-1)$ times: at each step we apply Remark 4.1 to the function $\Phi_{i}$, that is the restriction of $\Phi$ to the $(n-i)$-dimensional sphere $S^{n-1} \cap\left(\boldsymbol{v}_{1}^{*}\right)^{\perp} \cap \cdots \cap\left(\boldsymbol{v}_{i-1}^{*}\right)^{\perp}$. In this way we get $(n-1)$ vectors $\boldsymbol{v}_{1}^{*}, \ldots, \boldsymbol{v}_{n-1}^{*}$ which are orthonormal and for which 4.4) holds 
(with $\boldsymbol{v}_{1}^{*}$ replaced by $\boldsymbol{v}_{i}^{*}, 1 \leqslant i \leqslant n-1$ ). After some orthogonal transformation, we may assume $\boldsymbol{v}_{i}^{*}=\boldsymbol{e}_{i}$, for $i=1, \ldots, n-1$.

To prove Theorems 1.14 and 1.19 we will use an argument with some flavor of "integral geometry". The use of a integral geometry approach for the study of anisotropic nonlocal perimeter functionals turns out to be useful also in the recent paper of Ludwig [30].

Let us introduce some notation. In the the sequel $L \subset \mathbb{R}^{n}$ denotes a linear subspace with dimension $m$ with $1 \leqslant m \leqslant n-1$. We let $\left\{\boldsymbol{v}_{i}\right\}_{1 \leqslant i \leqslant m}$ be a orthonormal basis of $L$ and denote

$$
L^{\perp}=\left\{y: \boldsymbol{v}_{i} \cdot y=0 \text { for all } 1 \leqslant i \leqslant m\right\} .
$$

Let $\Omega \subset \mathbb{R}^{n}$ be a bounded open set. Given a set $E$ with finite perimeter in $\Omega$, let $u=\chi_{E}$. Note that the distributional gradient $\nabla u$ is a vector valued measure in $B_{1}$. We will denote $\nabla_{L} u$ the projected (vector valued) measure

$$
\nabla_{L} u=\sum_{i=1}^{m}\left(\nabla u \cdot \boldsymbol{v}_{i}\right) \boldsymbol{v}_{i}
$$

For each (almost every) $y \in L^{\perp}$ we denote $I_{E, \Omega}(L, y)$ the total variation of $u=\chi_{E}$ restricted to $(y+L) \cap \Omega$. That is, we define

$$
I_{E, \Omega}(L, y):=\sup \left\{-\int_{(y+L) \cap \Omega} u(z) \operatorname{div} \phi(z) d H^{m}(z): \phi \in C_{c}^{1}\left((y+L) \cap \Omega ; L \cap B_{1}\right)\right\} .
$$

Sometimes, when $E$ and $\Omega$ are fixed and there is no misunderstanding, for the sake of simplicity we will also use the notation

$$
I(L, y):=I_{E, \Omega}(L, y) .
$$

When $m=1$ and $L=\mathbb{R} \boldsymbol{v}$ for some $\boldsymbol{v} \in S^{n-1}$ we will also denote $I(L, y)$ as $I(\boldsymbol{v}, y)$. In the case $m=1$ we define also $I(\boldsymbol{v}, y)_{+}$and $I(\boldsymbol{v}, y)_{-}$respectively as

$$
I(\boldsymbol{v}, y)_{ \pm}:=\sup \left\{\mp \int_{(y+\mathbb{R} \boldsymbol{v}) \cap \Omega} u(z) \phi^{\prime}(z) d H^{1}(z): \phi \in C_{c}^{1}((y+\mathbb{R} \boldsymbol{v}) \cap \Omega ;[0,1])\right\},
$$

where $\phi^{\prime}=\partial_{\boldsymbol{v}} \phi$ denotes the tangential derivative along the (oriented) line $y+\mathbb{R} \boldsymbol{v}$. This auxiliary function $I(\boldsymbol{v}, y)_{ \pm}$is useful to detect the monotonicity of $\chi_{E}$, as pointed out in the following result:

Lemma 4.3. Let $E$ be a set of finite perimeter in a convex open set $\Omega, \boldsymbol{v} \in S^{n-1}$ and $y \in \boldsymbol{v}^{\perp}$. Then:

(i) If $I(\boldsymbol{v}, y)_{+}=0$, then $\chi_{E}$ restricted to $(y+\mathbb{R} \boldsymbol{v}) \cap \Omega$ is nonincreasing;

(ii) If $I(\boldsymbol{v}, y)_{-}=0$, then $\chi_{E}$ restricted to $(y+\mathbb{R} \boldsymbol{v}) \cap \Omega$ is nondecreasing;

(iii) If $I(\boldsymbol{v}, y)=0$, then $(y+\mathbb{R} \boldsymbol{v}) \cap \Omega$ is contained either in $E$ or in $\mathcal{C} E$. 
Proof. To prove (i), we denote $(a, b) \subset \mathbb{R}$ the open interval $\{t \in \mathbb{R}: y+t \boldsymbol{v} \in \Omega\}$ - here we use the convexity of $\Omega$. Let us define $\tilde{u}(t):=\chi_{E}(y+t \boldsymbol{v})$ and we remark that $\tilde{u}$ is of bounded variation in $[a, b]$ - see e.g. Corollary 6.9 of [1] or Theorem 2 in Section 5.10 .2 of [23]. Then, given any $\phi \in C_{c}^{1}((y+\mathbb{R} \boldsymbol{v}) \cap \Omega ;[0,1])$, we define $\tilde{\phi}(t):=\varphi(y+t \boldsymbol{v})$ and we use 4.6 to find that

$$
0=I(\boldsymbol{v}, y)_{+} \geqslant-\int_{a}^{b} u(y+t \boldsymbol{v}) \phi^{\prime}(y+t \boldsymbol{v}) d t=-\int_{a}^{b} \tilde{u}(t) \tilde{\phi}^{\prime}(t) d t
$$

for all $\tilde{\phi} \in C_{c}^{1}((a, b) \cap \Omega ;[0,1])$. As a consequence (see e.g. Corollary 9.91 in [40]), we have that $\tilde{u}$ is nonincreasing, which is (i).

The proof of (ii) is analogous. Now we prove (iii). By taking $\phi$ identically zero in $(4.6)$, we see that $I(\boldsymbol{v}, y)_{ \pm} \geqslant 0$. Therefore, if $I(\boldsymbol{v}, y)=0$, then $I(\boldsymbol{v}, y)_{+}=$ $I(\boldsymbol{v}, y)_{-}=0$, and thus we can use (i) and (ii) to deduce that $\chi_{E}$ restricted to $y+\mathbb{R} \boldsymbol{v}$ is constant, which gives (iii).

The following proposition gives equivalent formulas to compute the total variation of the projection of onto some linear subspace $L$ of the measure $\nabla u, u$ being the characteristic function of a set of finite perimeter.

Proposition 4.4. Let $\Omega \subset \mathbb{R}^{n}$ be a bounded open set, $E$ be a set of finite perimeter in $\Omega$, and $u=\chi_{E}$. Let $L \subset \mathbb{R}^{n}$ be linear subspace with dimension $m$ with $1 \leqslant m \leqslant n-1$. We let $\left\{\boldsymbol{v}_{i}\right\}_{1 \leqslant i \leqslant m}$ be a orthonormal basis of $L$.

Then, $I_{E, \Omega}(L, y) \geqslant 0$ is measurable in the variable $y \in L^{\perp}$ and the following identities hold

$$
\begin{aligned}
\left|\nabla_{L} u\right|(\Omega): & =\sup \left\{-\int_{\Omega} u(x) \operatorname{div} \phi(x) d x: \phi \in C_{c}^{1}\left(\Omega ; L \cap B_{1}\right)\right\} \\
& =\int_{\partial^{*} E \cap \Omega} \sqrt{\sum_{i=1}^{m}\left(\boldsymbol{v}_{i} \cdot \nu_{E}(x)\right)^{2}} d H^{n-1}(x) \\
& =\int_{L^{\perp}} I_{E, \Omega}(L, y) d H^{n-m}(y)
\end{aligned}
$$

Moreover if $m=1$ and $L=\mathbb{R} \boldsymbol{v}$ then

$$
\begin{gathered}
\left|\partial_{\boldsymbol{v}} u\right|(\Omega)=\int_{\partial^{*} E \cap \Omega}\left|\boldsymbol{v} \cdot \nu_{E}(x)\right| d H^{n-1}(x)=\int_{\boldsymbol{v}^{\perp}} I_{E, \Omega}(\boldsymbol{v}, y) d H^{n-1}(y), \\
\left(\partial_{\boldsymbol{v}} u\right)_{ \pm}(\Omega)=\int_{\partial^{*} E \cap \Omega}\left(-\boldsymbol{v} \cdot \nu_{E}(x)\right)_{ \pm} d H^{n-1}(x)=\int_{\boldsymbol{v}^{\perp}} I_{E, \Omega}(\boldsymbol{v}, y)_{ \pm} d H^{n-1}(y),
\end{gathered}
$$

and for a.e. $y \in \boldsymbol{v}^{\perp}$ we have

$$
\begin{gathered}
I_{E, \Omega}(\boldsymbol{v}, y)=H^{0}\left(\partial^{*} E \cap \Omega \cap(y+\mathbb{R} \boldsymbol{v})\right) \\
I_{E, \Omega}(\boldsymbol{v}, y)_{ \pm}=H^{0}\left(\left\{x \in \partial^{*} E \cap \Omega \cap(y+\mathbb{R} \boldsymbol{v}): \mp \boldsymbol{v} \cdot \nu_{E}(x)>0\right\}\right) .
\end{gathered}
$$


The proof of Proposition 4.4 relies on standard results from the theory of sets of finite perimeter and functions of bounded variation (see [31, 23]), and will be sketched in the Appendix. Note that if $\partial E$ has smooth boundary in $B_{1}$ then the proof of Proposition 4.4 is rather elementary. For related results for $m=1$ in the context of integral geometry formulae for sets of finite perimeter see also [30, Section 1.1] and [45, Theorem 1].

The well-known Cauchy-Crofton formula (and indeed a generalized version of it) can be obtained as a corollary of the previous proposition with $m=1$, as pointed out by the next result:

Corollary 4.5. Let $E$ be a set of finite perimeter in $B_{1}$ and $\boldsymbol{v} \in S^{n-1}$. Let $\boldsymbol{v}^{\perp}$ denote the hyperplane $\{y: \boldsymbol{v} \cdot y=0\}$.

Then

$$
\operatorname{Per}_{\Omega}(E)=c \int_{S^{n-1}} d H^{n-1}(\boldsymbol{v}) \int_{\boldsymbol{v}^{\perp}} d H^{n-1}(y) H^{0}\left(\partial^{*} E \cap \Omega \cap(y+\mathbb{R} \boldsymbol{v})\right)
$$

where $H^{0}\left(\partial^{*} E \cap \Omega \cap(y+\mathbb{R} \boldsymbol{v})\right)$ counts the number of intersections inside $\Omega$ of the line $y+\mathbb{R} \boldsymbol{v}$ with the reduced boundary of $E$. The constant $c=c(n)$ is given by

$$
c=\left(\int_{S^{n-1}}|\boldsymbol{v} \cdot \boldsymbol{w}| d H^{n-1}(\boldsymbol{v})\right)^{-1}
$$

where $\boldsymbol{w} \in S^{n-1}$ is any fixed unit vector - this value does not depend on $\boldsymbol{w}$.

Proof. Using 4.8) and (4.10), we have

$$
\int_{\partial^{*} E \cap \Omega}\left|\boldsymbol{v} \cdot \nu_{E}(x)\right| d H^{n-1}(x)=\int_{\boldsymbol{v}^{\perp}} H^{0}\left(\partial^{*} E \cap \Omega \cap(y+\mathbb{R} \boldsymbol{v})\right) d H^{n-1}(y) .
$$

The corollary follows integrating with respect to $\boldsymbol{v} \in S^{n-1}$.

The following observation will be crucial in the proof of our Theorems 1.14 and 1.19 .

Remark 4.6. When $m=1$, for a.e. $y \in \boldsymbol{v}^{\perp}$,

$$
I(\boldsymbol{v}, y), I(\boldsymbol{v}, y)_{+} \text {and } I(\boldsymbol{v}, y)_{-} \text {are nonnegative integers. }
$$

Indeed this follows from 4.10 and 4.11) since $H^{0}$ is the counting measure.

In the rest of this section we will consider the functions

$$
\Phi_{+}(\boldsymbol{v}):=\left(\partial_{\boldsymbol{v}} u\right)_{+}\left(B_{1}\right) \text { and } \Phi_{-}(\boldsymbol{v}):=\left(\partial_{\boldsymbol{v}} u\right)_{-}\left(B_{1}\right),
$$

where $u=\chi_{E}$ is the characteristic function of a set $E$ of finite perimeter in $B_{1}$. By (4.9), we have

$$
\Phi_{ \pm}(\boldsymbol{v})=\int_{\boldsymbol{v}^{\perp}} I_{E, B_{1}}(\boldsymbol{v}, y)_{ \pm} d H^{n-1}(y) .
$$

With this observation, we can reformulate Lemma 4.3 in this way:

Lemma 4.7. Let $E$ be a set of finite perimeter in $B_{1}, \boldsymbol{v} \in S^{n-1}$ and $\mu \geqslant 0$. Then: 
(i) If $\Phi_{+}(\boldsymbol{v}) \leqslant \mu\left(\right.$ resp. $\left.\Phi_{-}(\boldsymbol{v}) \leqslant \mu\right)$, then there exists $\mathcal{B} \subseteq \boldsymbol{v}^{\perp}$ with $H^{n-1}(\mathcal{B}) \leqslant \mu$ and such that for any $y \in \boldsymbol{v}^{\perp} \backslash \mathcal{B}$ we have that $\chi_{E}$ restricted to $(y+\mathbb{R} \boldsymbol{v}) \cap B_{1}$ is nonincreasing (resp. nondecreasing);

(ii) If $\max \left\{\Phi_{+}(\boldsymbol{v}), \Phi_{-}(\boldsymbol{v})\right\} \leqslant \mu$, then there exists $\mathcal{B} \subseteq \boldsymbol{v}^{\perp}$ with $H^{n-1}(\mathcal{B}) \leqslant \mu$ and such that for any $y \in \boldsymbol{v}^{\perp} \backslash \mathcal{B}$ we have that $(y+\mathbb{R} \boldsymbol{v}) \cap B_{1}$ is contained either in $E$ or in $\mathcal{C} E$.

Proof. Since (ii) follows from (i), we focus on the proof of (i) and we suppose that $\Phi_{+}(\boldsymbol{v}) \leqslant \mu$ (the case $\Phi_{-}(\boldsymbol{v}) \leqslant \mu$ is analogous). We set

$$
\mathcal{B}:=\left\{y \in \boldsymbol{v}^{\perp}: I_{E, B_{1}}(\boldsymbol{v}, y)_{+} \neq 0\right\} .
$$

By Remark 4.6, we have that

$$
\mathcal{B}=\left\{y \in \boldsymbol{v}^{\perp}: I_{E, B_{1}}(\boldsymbol{v}, y)_{+} \geqslant 1\right\}
$$

and therefore, by 4.13 ,

$$
\mu \geqslant \Phi_{-}(\boldsymbol{v})=\int_{\mathcal{B}} I_{E, B_{1}}(\boldsymbol{v}, y)_{+} d H^{n-1}(y) \geqslant H^{n-1}(\mathcal{B})
$$

which is the desired estimate on $\mathcal{B}$.

Notice that, by construction, if $y \in \boldsymbol{v}^{\perp} \backslash \mathcal{B}$, then $I_{E, B_{1}}(\boldsymbol{v}, y)_{+}=0$, and so Lemma 4.3 gives that $\chi_{E}$ restricted to $(y+\mathbb{R} \boldsymbol{v}) \cap \Omega$ is nonincreasing, as desired.

With this, we obtain the following flatness result:

Lemma 4.8. Let $E$ be a set of finite perimeter in $B_{1}, u=\chi_{E}, \boldsymbol{v} \in S^{n-1}$ and $\Phi_{ \pm}$ be as in 4.12).

Suppose that for all $\boldsymbol{v} \in S^{n-1}$,

$$
\min \left\{\Phi_{+}(\boldsymbol{v}), \Phi_{-}(\boldsymbol{v})\right\} \leqslant \mu
$$

for some $\mu>0$. Then, after some rotation the set $E$ satisfies $(\mathbf{F} \mathbf{1}),(\mathbf{F} 2)$, and $(\mathbf{F} \mathbf{3})$ on page 14, with

$$
\varepsilon=C(n) \mu
$$

where $C(n)$ is a constant depending only on the dimension.

Proof. We first observe that, since $E$ has finite perimeter in $B_{1}$, for $u=\chi_{E}, \nabla u$ is a vector valued measure and

$$
\Phi_{ \pm}(\boldsymbol{v})=\left(\partial_{\boldsymbol{v}} u\right)_{ \pm}\left(B_{1}\right)=(\nabla u \cdot \boldsymbol{v})_{ \pm}\left(B_{1}\right) .
$$

Then,

$$
\Phi_{ \pm}(-\boldsymbol{v})=\Phi_{\mp}(\boldsymbol{v})
$$

In addition, we have

$$
\left|\Phi_{+}(\boldsymbol{v})-\Phi_{+}(\boldsymbol{w})\right| \leqslant|\boldsymbol{v}-\boldsymbol{w}||\nabla u|\left(B_{1}\right)
$$

and same holds for $\Phi_{-}$. Hence, in particular, $\Phi_{+}$and $\Phi_{-}$are continuous functions on $S^{n-1}$ satisfying the assumptions of Lemma 4.2 . 
Therefore, after some rotation we have

$$
\max \left\{\Phi_{+}\left(\boldsymbol{e}_{i}\right), \Phi_{-}\left(\boldsymbol{e}_{\boldsymbol{i}}\right)\right\} \leqslant \mu, \quad \text { for } 1 \leqslant i \leqslant n-1
$$

In addition, by (4.14), and possibly changing $\boldsymbol{e}_{n}$ by $-\boldsymbol{e}_{n}$, we may assume that

$$
\Phi_{+}\left(\boldsymbol{e}_{n}\right) \leqslant \mu .
$$

Using (4.15) and Lemma 4.7 we conclude that, for any $i \in\{1, \ldots, n-1\}$, there exists $\mathcal{B}_{i} \subseteq \boldsymbol{e}_{i}^{\perp}$, with $H^{n-1}\left(\mathcal{B}_{i}\right) \leqslant \mu$, and such that for any $y \in \boldsymbol{e}_{i}^{\perp} \backslash \mathcal{B}_{i}$ we have that

$$
\left(y+\mathbb{R} \boldsymbol{e}_{i}\right) \cap B_{1} \text { is contained either in } E \text { or in } \mathcal{C} E \text {. }
$$

Similarly, by (4.16) and Lemma 4.7, we see that there exists $\mathcal{B}_{n} \subseteq \boldsymbol{e}_{n}^{\perp}$, with $H^{n-1}\left(\mathcal{B}_{n}\right) \leqslant$ $\mu$, and such that for any $y \in \boldsymbol{e}_{n}^{-} \backslash \mathcal{B}_{n}$ we have that

$$
\chi_{E} \text { restricted to }\left(y+\mathbb{R} \boldsymbol{e}_{n}\right) \cap B_{1} \text { is nonincreasing. }
$$

Notice that (4.18) implies (F2). Now we complete the proof of the desired result in three steps: first, we establish $(\mathbf{F 1})$ in the two-dimensional case, then in the three-dimensional case, and finally we prove $(\mathbf{F} 3)$.

Step 1. Let us show that (F1) holds for $\varepsilon=2 \mu$ first in dimension $n=2$. Let us assume that $\mu<2$ since otherwise $2 \mu>\pi 1^{2}=\left|B_{1}\right|$ and there is nothing to prove.

By (4.17), for any $t$ outside the small set $\mathcal{B}_{1}$,

$$
\text { the segment }\left\{x_{2}=t\right\} \cap B_{1} \text { is either contained in } E \text { or in } \mathcal{C} E \text {; }
$$

here, we are identifying points $y \in \boldsymbol{e}_{1}^{\perp}$ and points $t \in(-1,1)$ via $y=(0, t)$.

Therefore, we can define $\mathcal{G}_{E}$ (resp., $\mathcal{G}_{\mathcal{C} E}$ ) as the family of $t \in(-1,1)$ for which $\left\{x_{2}=\right.$ $t\} \cap B_{1}$ is contained in $E$ (resp., in $\mathcal{C} E$ ), and then 4.19 says that

$$
(-1,1)=\mathcal{G}_{E} \cup \mathcal{G}_{\mathcal{C} E} \cup \mathcal{B}_{1}
$$

The fact that $\chi_{E}$ is nonincreasing along the vertical direction for a (nonvoid) set of vertical segments (as warranted by (4.18)), implies that the sets $\mathcal{G}_{E}$ and $\mathcal{G}_{\mathcal{C} E}$ are ordered with respect to the vertical direction. More precisely, there exist $t_{*}, t^{*} \in$ $[-1,1]$, such that

$$
\operatorname{ess} \sup \mathcal{G}_{E}=t_{*} \leqslant t^{*}=\operatorname{ess} \inf \mathcal{G}_{\mathcal{C} E} .
$$

This implies that, for all $t \in\left[t_{*}, t^{*}\right]$,

$$
\begin{aligned}
\left|\left(E \backslash\left\{x_{2} \leqslant t\right\}\right) \cap B_{1}\right|+\left|\left(\left\{x_{2} \leqslant t\right\} \backslash E\right) \cap B_{1}\right| & \leqslant\left|\left\{x_{2} \in \mathcal{B}_{1}\right\} \cap B_{1}\right| \\
& \leqslant 2\left|\mathcal{B}_{1}\right| \leqslant 2 \mu
\end{aligned}
$$

and thus (F1) follows.

In dimension $n=2$ we can obtain an even stronger information since

$$
E \cap\left\{x_{1} \notin \mathcal{B}_{2}\right\} \cap B_{1} \supset\left\{x_{2} \leqslant t_{*}\right\} \cap\left\{x_{1} \notin \mathcal{B}_{2}\right\} \cap B_{1}
$$

and

$$
\mathcal{C} E \cap\left\{x_{1} \notin \mathcal{B}_{2}\right\} \cap B_{1} \supset\left\{x_{2} \geqslant t^{*}\right\} \cap\left\{x_{1} \notin \mathcal{B}_{2}\right\} \cap B_{1}
$$


Therefore, there exits $g: B_{1}^{(n-1)} \rightarrow[-1,1]$ such that

$$
E \cap\left\{x_{1} \notin \mathcal{B}_{2}\right\} \cap B_{1}=\left\{x_{2} \leqslant g\left(x_{1}\right)\right\} \cap\left\{x_{1} \notin \mathcal{B}_{2}\right\}
$$

with the oscillation of $g$ bounded by $\left(t^{*}-t_{*}\right) \leqslant H^{1}(\mathcal{B}) \leqslant \mu$ (see Figure 5 ).

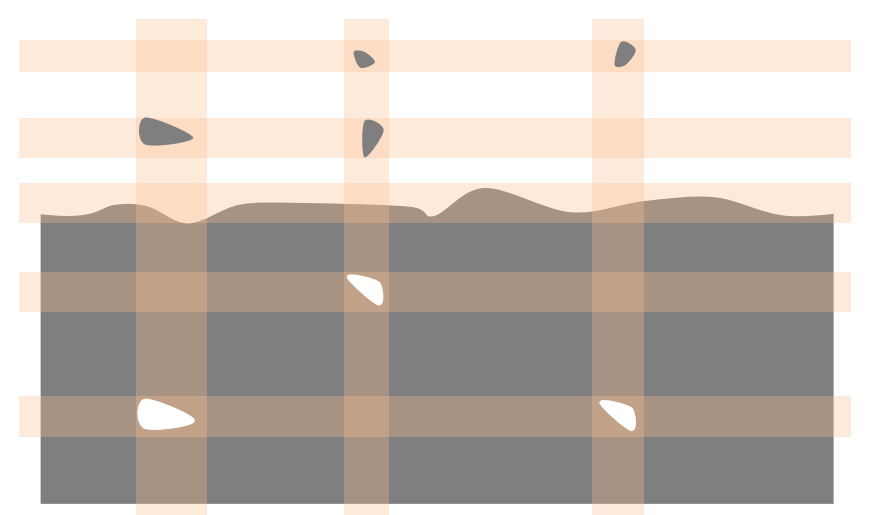

Figure 5. The two-dimensional picture.

Step 2. Let us show that (F1) holds for $\varepsilon=C(n) \mu$ in dimensions $n \geqslant 3$. For this, we define $L=\boldsymbol{e}_{n}^{\perp}$ and we use (4.7) and (4.8) in Proposition 4.4 to estimate

$$
\begin{aligned}
\left|\nabla_{L} u\right|\left(B_{1}\right) & =\int_{\partial^{*} E \cap B_{1}} \sqrt{\sum_{i=1}^{n-1}\left(\boldsymbol{e}_{i} \cdot \nu_{E}(x)\right)^{2}} d H^{n-1}(x) \\
& \leqslant \frac{1}{\sqrt{n-1}} \sum_{i=1}^{n-1} \int_{\partial^{*} E \cap B_{1}}\left|\boldsymbol{e}_{i} \cdot \nu_{E}(x)\right| d H^{n-1}(x) \\
& =\frac{1}{\sqrt{n-1}} \sum_{i=1}^{n-1}\left|\partial_{\boldsymbol{e}_{i}} u\right|\left(B_{1}\right) .
\end{aligned}
$$

Now we observe that, by 4.12 and 4.15),

$$
\left|\partial_{\boldsymbol{e}_{i}} u\right|\left(B_{1}\right)=\left(\partial_{\boldsymbol{e}_{i}} u\right)_{+}\left(B_{1}\right)+\left(\partial_{\boldsymbol{e}_{i}} u\right)_{-}\left(B_{1}\right)=\Phi_{+}\left(\boldsymbol{e}_{i}\right)+\Phi_{-}\left(\boldsymbol{e}_{i}\right) \leqslant 2 \mu,
$$

which, together with 4.20 , gives that

$$
\left|\nabla_{L} u\right|\left(B_{1}\right) \leqslant 2 \sqrt{n-1} \mu .
$$

Moreover, we note that there exists a small constant $\bar{\mu}=\bar{\mu}(n)>0$-depending only on $n$ - such that for $\mu \in(0, \bar{\mu})$ and $r=\mu^{\frac{1}{n+1}}$ we have

$$
\mu \leqslant c \mu^{\frac{n-1}{n+1}} \leqslant \frac{1}{4} H^{n-1}\left(B_{r}^{(n-1)}\right) .
$$

We now use that $I_{E, B_{1}}(L, y)$ is the relative perimeter of $(y+L) \cap E$ in the $(n-1)$ dimensional ball $(y+L) \cap B_{1}$-recall (4.5). Thus, using the relative isoperimetric 
inequality at each horizontal slice $B_{1} \cap\left\{x_{n}=t\right\}$ we find that

$$
\begin{aligned}
\min \left\{H^{n-1}\left(E \cap B_{1} \cap\left\{x_{n}=t\right\}\right), H^{n-1}\left(\mathcal{C} E \cap B_{1} \cap\left\{x_{n}=t\right\}\right)\right\} \\
\leqslant C \min \left\{1, I_{E, B_{1}}(L, y)^{\frac{n-1}{n-2}}\right\} \leqslant \bar{C} I_{E, B_{1}}(L, y),
\end{aligned}
$$

where $\bar{C}>0$ is a suitable constant (depending only on $n$ ) and the last coordinate of $y$ equals to $t$.

Let us define the "horizontal bad set" as

$$
\mathcal{B}:=\mathcal{B}^{\prime} \cup \mathcal{B}^{\prime \prime}
$$

where

$$
\begin{aligned}
\mathcal{B}^{\prime} & :=\left\{t \in(-1,1):|t| \geqslant \sqrt{1-r^{2}}\right\} \\
\text { and } \quad \mathcal{B}^{\prime \prime} & :=\left\{t \in(-1,1):|t|<\sqrt{1-r^{2}} \text { and } \bar{C} I_{E, B_{1}}(L, y)>\bar{\mu}\right\} .
\end{aligned}
$$

We also define $\mathcal{G}_{E}$ as the family of $t \in(-1,1)$ for which $|t|<\sqrt{1-r^{2}}$ and

$$
H^{n-1}\left(\left\{x_{n}=t\right\} \cap B_{1} \cap \mathcal{C} E\right) \leqslant \mu .
$$

Similarly, we define $\mathcal{G}_{\mathcal{C} E}$ as the family of $t \in(-1,1)$ for which $|t|<\sqrt{1-r^{2}}$ and

$$
H^{n-1}\left(\left\{x_{n}=t\right\} \cap B_{1} \cap E\right) \leqslant \mu .
$$

By(4.23), it follows that

$$
(-1,1) \backslash \mathcal{B} \subseteq \mathcal{G}_{E} \cup \mathcal{G}_{\mathcal{C} E}
$$

In addition, by 4.7) and (4.21),

$$
2 \sqrt{n-1} \mu \geqslant\left|\nabla_{L} u\right|\left(B_{1}\right) \geqslant \int_{\mathcal{B}^{\prime \prime}} I_{E, \Omega}(L, y) d H^{1}(y) \geqslant \frac{\bar{\mu}}{\bar{C}} H^{1}\left(\mathcal{B}^{\prime \prime}\right) .
$$

Therefore $H^{1}\left(\mathcal{B}^{\prime \prime}\right) \leqslant C_{0} \mu$ and then

$$
H^{n}\left(\left\{\left(x^{\prime}, t\right) \in B_{1}: t \in \mathcal{B}^{\prime \prime}\right\}\right) \leqslant C_{1} H^{1}\left(\mathcal{B}^{\prime \prime}\right) \leqslant C_{2} \mu,
$$

for some constants $C_{0}, C_{1}, C_{2}>0$.

Furthermore, if $\left(x^{\prime}, t\right) \in B_{1}$ and $t \in \mathcal{B}^{\prime}$, then

$$
\left|x^{\prime}\right|^{2}=\left|x^{\prime}\right|^{2}+t^{2}-t^{2} \leqslant 1-\left(1-r^{2}\right)=r^{2},
$$

which implies that $x^{\prime} \in B_{r}^{(n-1)}$, and so that

$$
H^{n}\left(\left\{\left(x^{\prime}, t\right) \in B_{1}: t \in \mathcal{B}^{\prime}\right\}\right) \leqslant C_{3} r^{n-1}\left(1-\sqrt{1-r^{2}}\right) \leqslant C_{4} r^{n+1}=C_{4} \mu,
$$

for some $C_{3}, C_{4}>0$. This and 4.27 give that

$$
H^{n}\left(\left\{\left(x^{\prime}, t\right) \in B_{1}: t \in \mathcal{B}\right\}\right) \leqslant C_{5} \mu,
$$

for some $C_{5}>0$.

Now we claim that the sets $\mathcal{G}_{E}$ and $\mathcal{G}_{\mathcal{C} E}$ are ordered with respect to the vertical direction, namely there exist $t_{*}, t^{*} \in[-1,1]$, such that

$$
\operatorname{ess} \sup \mathcal{G}_{E}=t_{*} \leqslant t^{*}=\operatorname{ess} \inf \mathcal{G}_{\mathcal{C} E}
$$


For this, let $t_{1}<t_{2} \in(-1,1) \backslash \mathcal{B}$. We show that

$$
\text { if } t_{1} \in \mathcal{G}_{\mathcal{C} E} \text { then } t_{2} \in \mathcal{G}_{\mathcal{C} E} \text {. }
$$

We argue by contradiction, assuming that $t_{2} \notin \mathcal{G}_{\mathcal{C} E}$. Then, by $(4.26)$, we obtain that $t_{2} \in \mathcal{G}_{E}$. Consequently, by (4.24), we have that $t_{2}^{2}<1-r^{2}$ and

$$
H^{n-1}\left(\left\{x_{n}=t_{2}\right\} \cap B_{1} \cap \mathcal{C} E\right) \leqslant \mu .
$$

We can write this as $\chi_{E}\left(x^{\prime}, t_{2}\right)=1$ for any $x^{\prime}$ in the ball $B_{\sqrt{1-t_{2}^{2}}}^{(n-1)}$ outside a set of $(n-1)$-dimensional measure less than $\mu$ (so, in particular, for any $x^{\prime}$ in the smaller ball $B_{r}^{(n-1)}$ outside a set of $(n-1)$-dimensional measure less than $\left.\mu\right)$.

Also, the condition $t_{1} \in \mathcal{G}_{\mathcal{C} E}$ and 4.25 give that $t_{1}^{2}<1-r^{2}$ and

$$
H^{n-1}\left(\left\{x_{n}=t_{1}\right\} \cap B_{1} \cap E\right) \leqslant \mu .
$$

We can write this as $\chi_{E}\left(x^{\prime}, t_{1}\right)=0$ for any $x^{\prime} \in B_{\sqrt{1-t_{1}^{2}}}^{(n-1)}$ outside a set of $(n-1)$ dimensional measure less than $\mu$ (so, in particular, for any $x^{\prime} \in B_{r}^{(n-1)}$ outside a set of $(n-1)$-dimensional measure less than $\mu)$.

By 4.18), we also know that $\chi_{E}\left(x^{\prime}, t\right)$ is nonincreasing in $t$ outside $\mathcal{B}_{n}$, which is another set of $(n-1)$-dimensional measure less than $\mu$.

This means that, for $x^{\prime} \in B_{r}^{(n-1)}$ outside a set of $(n-1)$-dimensional measure less than $3 \mu$, we have that

$$
1=\chi_{E}\left(x^{\prime}, t_{2}\right) \leqslant \chi_{E}\left(x^{\prime}, t_{1}\right)=0 .
$$

We stress the fact that this set to which $x^{\prime}$ belongs is nonvoid, since $\left.H^{n-1} B_{r}^{(n-1)}\right)$ is strictly bigger than $3 \mu$, thanks to 4.22 . Therefore, the inequality in 4.30 provides a contradiction. This proves (4.29). Similarly, one proves that

$$
\text { if } t_{2} \in \mathcal{G}_{E} \text { then } t_{1} \in \mathcal{G}_{E} \text {. }
$$

By putting together (4.29) and (4.31), one obtains (4.28).

Then it readily follows that $(\mathbf{F 1})$ is satisfied with $\varepsilon=C(n) \mu$.

Step 3 We show that (F3) with $\varepsilon=\mu$ in any dimension $n \geqslant 2$.

Recall that we denote $F^{\varepsilon}=\left\{\left(x^{\prime}, x_{n} / \varepsilon\right):\left(x^{\prime}, x_{n}\right) \in F\right\}$. Using Proposition 4.4 we estimate

$$
\begin{aligned}
\operatorname{Per}_{B_{1}^{\varepsilon}}\left(E^{\varepsilon}\right)= & \sup \left\{-\int_{B_{1}^{\varepsilon}} \chi_{E^{\varepsilon}} \operatorname{div} \phi d x: \phi \in C_{c}^{1}\left(B_{1}^{\varepsilon} ; \mathbb{R}^{n}\right),|\phi| \leqslant 1\right\} \\
\leqslant & \sum_{i=1}^{n-1} \sup \left\{-\int_{B_{1}^{\varepsilon}} \chi_{E^{\varepsilon}} \partial_{i} \psi d x: \psi \in C_{c}^{1}\left(B_{1}^{\varepsilon}\right),|\psi| \leqslant 1\right\}+ \\
& \quad+\sup \left\{-\int_{B_{1}^{\varepsilon}} \chi_{E^{\varepsilon}} \partial_{n} \psi d x: \psi \in C_{c}^{1}\left(B_{1}\right),|\psi| \leqslant 1\right\}
\end{aligned}
$$


Then, using the change of variables $y^{\prime}=x^{\prime}$ and $y_{n}=\varepsilon x_{n}$, we have

$$
\begin{aligned}
\operatorname{Per}_{B_{1}^{\varepsilon}}\left(E^{\varepsilon}\right) \leqslant & \sum_{i=1}^{n-1} \sup \left\{-\int_{B_{1}} \chi_{E} \partial_{i} \bar{\psi} \frac{d x}{\varepsilon}: \bar{\psi} \in C_{c}^{1}\left(B_{1}\right),|\bar{\psi}| \leqslant 1\right\}+ \\
& \quad+\sup \left\{-\int_{B_{1}} \chi_{E} \varepsilon \partial_{n} \bar{\psi} \frac{d x}{\varepsilon}: \bar{\psi} \in C_{c}^{1}\left(B_{1}\right),|\bar{\psi}| \leqslant 1\right\} \\
\leqslant & \frac{2}{\varepsilon} \sum_{i=1}^{n-1} \max \left\{\Phi_{+}\left(\boldsymbol{e}_{i}\right), \Phi_{-}\left(\boldsymbol{e}_{i}\right)\right\}+ \\
& +\min \left\{\Phi_{+}\left(\boldsymbol{e}_{n}\right), \Phi_{-}\left(\boldsymbol{e}_{n}\right)\right\}+2\left|\Phi_{+}\left(\boldsymbol{e}_{n}\right)-\Phi_{-}\left(\boldsymbol{e}_{n}\right)\right|
\end{aligned}
$$

Now we use that

$$
\Phi_{+}\left(\boldsymbol{e}_{n}\right)-\Phi_{-}\left(\boldsymbol{e}_{n}\right)=\int_{B_{1}} \partial_{\boldsymbol{e}_{n}} u=\int_{\partial B_{1}} u(x) \nu_{n}(x) d H^{n-1}(x) .
$$

Hence,

$$
\left|\Phi_{+}\left(\boldsymbol{e}_{n}\right)-\Phi_{-}\left(\boldsymbol{e}_{n}\right)\right| \leqslant \int_{\partial B_{1}}\left|\nu_{n}(x)\right| d H^{n-1}(x)=2\left|B_{1}^{(n-1)}\right| .
$$

Thus, taking $\varepsilon=\mu$ and using 4.15) and 4.16), we obtain

$$
\operatorname{Per}_{B_{1}^{\varepsilon}}\left(E^{\varepsilon}\right) \leqslant 2(n-1)+\varepsilon+4\left|B_{1}^{(n-1)}\right| \leqslant c(n)
$$

and (F3) follows.

We now give the

Proof of Theorem 1.19. For the proof we need to combine Lemmas 2.1, 2.4, 2.5 and 4.8 .

More precisely, using Lemma 2.1, point (a), and Lemma 2.4 we find that for any $\varepsilon>0$ there exists $t_{0}$ such that for $t \in\left(0, t_{0}\right)$

$$
\min \left\{L_{K}\left(E_{R, t} \backslash E, E \backslash E_{R, t}\right), L_{K}\left(E_{R,-t} \backslash E, E \backslash E_{R,-t}\right)\right\} \leqslant(\eta / 4+\varepsilon) t^{2},
$$

where $E_{R, t}$ is defined as in (2.4) and

$$
\eta=\frac{32}{R^{2}} P_{K^{*}, B_{R}}(E)
$$

This implies that for all $\boldsymbol{v}$ there is some sequence $t_{k} \rightarrow 0, t_{k} \in(-1,1)$ such that

$$
\lim _{k \rightarrow \infty} t_{k}^{-2} L_{K}\left(E_{R, t_{k}} \backslash E, E \backslash E_{R, t_{k}}\right) \leqslant \eta / 4
$$

Now, by definition of $E_{R, t}$ we have

$$
E_{R, t} \cap B_{1}=(E+t \boldsymbol{v}) \cap B_{1} .
$$

and thus using the assumption $(1.6)$-i.e. $K \geqslant 1$ in $B_{2}$ - we obtain

$$
\lim _{k \rightarrow \infty} t_{k}^{-2}\left|\left(E+t_{k} \boldsymbol{v}\right) \backslash E\right| \cdot\left|E \backslash\left(E+t_{k} \boldsymbol{v}\right)\right| \leqslant \eta / 4
$$


Therefore, applying Lemma 2.5 we obtain

$$
\min \left\{\Phi_{+}(\boldsymbol{v}), \Phi_{-}(\boldsymbol{v})\right\} \leqslant \sqrt{\eta} / 2,
$$

where $\Phi_{ \pm}(\boldsymbol{v})=\left(-\partial_{\boldsymbol{v}} u\right)_{ \pm}\left(B_{1}\right)$.

Then, applying Lemma 4.8 we obtain that $E$ satisfies $(\mathbf{F 1}),(\mathbf{F} 2)$, and (F3) with

$$
\varepsilon=C(n) \sqrt{\eta}=C(n) \sqrt{\frac{P_{K^{*}, B_{R}}(E)}{R^{2}}} .
$$

The same inequality for $\varepsilon=\frac{C(n)}{\sqrt{\log R}} \sup _{\rho \in[1, R]} \sqrt{\frac{P_{K^{*}, B_{\rho}}(E)}{\rho^{2}}}$ is proved likewise using $\tilde{E}_{R, t}$ instead of $E_{R, t}$ and part (b) of Lemma 2.1 instead of part (a).

Theorem 1.14 and Corollaries 1.20, 1.21, 1.22 all follow by Theorem 1.19 and estimate for the quantity $P_{K^{*}, B_{R}}(E)$.

Proof of Theorem 1.14. Multiplying the kernel $K \in \mathcal{L}_{2}$ by a positive constant, we may assume that $\lambda \geqslant 2^{n+s}$ and hence $K$ satisfies $(1.3)-(1.7)$ with $K^{*}=C_{1} K$. Applying Corollary 1.8, we deduce that

$$
P_{K^{*}, B_{R}}(E)=C_{1} P_{K, B_{R}}(E) \leqslant C R^{n-s} \text {. }
$$

Thus, Theorem 1.14 follows by Theorem 1.19 and estimate 4.32 above.

Proof of Corollary 1.20. The proof follows by Theorem 1.19, after observing that if $K^{*} \in L^{1}\left(\mathbb{R}^{n}\right)$ and $E$ is a minimizer, then

$$
P_{K_{*}, B_{\rho}}(E) \leqslant\left|B_{\rho}\right| \int_{\mathbb{R}^{2}} K^{*}=\rho^{2}\left|B_{1}\right| \int_{\mathbb{R}^{2}} K^{*}
$$

Proof of Corollary 1.21. The proof follows by Theorem 1.19 and by Proposition 1.11.

Proof of Corollary 1.22. The proof follows by Corollary 1.21 using that for compactly supported kernels $K$, we have

$$
P_{K}\left(B_{R}\right) \sim R^{n-1}
$$

\section{EnERgy estimates With PeRTURBed KeRnels}

Lemma 5.1. Let $R_{0} \geqslant 1$. Assume that $K \geqslant 1$ in $B_{1}$. Let $Q=\left(-3 R_{0} / 2,3 R_{0} / 2\right)^{n}$ and $E \subset \mathbb{R}^{n}$ is measurable. Then,

$$
L_{K}(E \cap Q, \mathcal{C} E \cap Q) \geqslant c\left(n, R_{0}\right) \min \{|E \cap Q|,|\mathcal{C} E \cap Q|\} .
$$

Proof. Since the statement is invariant when we swap $E$ and $\mathcal{C} E$ we may assume $|E \cap Q| \leqslant|Q| / 2 \leqslant|\mathcal{C} E \cap Q|$.

Split $Q$ into a regular grid composed by $k^{n}$ open cubes of side $r=3 R_{0} / k$ with $r \in\left(n^{-1 / 2} / 8, n^{-1 / 2} / 4\right]$. We call these small cubes $Q_{i}, i \in I$. Let $\tilde{I}=\left\{i:\left|Q_{i} \cap E\right|>\right.$ $\left.\frac{1}{2}\left|Q_{i}\right|\right\}$. 
We have $\tilde{I} \neq I$ since $|E \cap Q| \leqslant|Q| / 2$. There are now two cases $\tilde{I}$ nonempty or empty.

On the one hand, if $\tilde{I}$ is nonempty then there are $i_{1} \in \tilde{I}$ and $i_{2} \in I \backslash \tilde{I}$ such that $Q_{i_{1}}$ and $Q_{i_{2}}$ are adjacent cubes. Then, since $r \leqslant n^{-1 / 2} / 4$ we have diam $\left(Q_{i}\right) \leqslant 1 / 2$ for all $i$ and thus $\operatorname{diam}\left(Q_{i_{1}} \cup Q_{i_{2}}\right) \leqslant 1$. Since $K \geqslant 1$ in $B_{1}$ we then have

$$
\begin{aligned}
L_{K}(E \cap Q, \mathcal{C} E \cap Q) & \geqslant L_{K}\left(E \cap Q_{i_{1}}, \mathcal{C} E \cap Q_{i_{2}}\right) \geqslant\left|E \cap Q_{i_{1}}\right| \cdot\left|\mathcal{C} E \cap Q_{i_{2}}\right| \\
& \geqslant \frac{1}{2}\left|Q_{i_{1}}\right| \cdot \frac{1}{2}\left|Q_{i_{2}}\right| \geqslant c(n) .
\end{aligned}
$$

On this case the estimate of the lemma follows since $|E \cap Q| \leqslant\left(3 R_{0}\right)^{n}$.

On the other hand, if $\tilde{I}$ is empty then $\left|Q_{i} \cap E\right| \leqslant \frac{1}{2}\left|Q_{i}\right|$ for all $i$ and

$$
\begin{aligned}
L_{K}(E \cap Q, \mathcal{C} E \cap Q) & \geqslant \sum_{i} L_{K}\left(E \cap Q_{i}, \mathcal{C} E \cap Q_{i}\right) \geqslant \sum_{i}\left|E \cap Q_{i}\right| \cdot \frac{1}{2}\left|Q_{i}\right| \\
& \geqslant c(n)|E \cap Q|,
\end{aligned}
$$

as desired.

Lemma 5.2. Let $R_{0} \geqslant 1$. Let $K$ be some kernel satisfying $K \geqslant 1$ in $B_{1}$. Let $E \subset \mathbb{R}^{n}$ measurable and $R \in 3 R_{0} \mathbb{N}$ and $Q_{R}=(-R / 2, R / 2)^{n}$. Denote $K_{0}(z)=\chi_{\left\{|z| \leqslant R_{0}\right\}}(z)$. Then,

$$
L_{K_{0}}\left(E \cap Q_{R}, \mathcal{C} E \cap Q_{R}\right) \leqslant C\left(n, R_{0}\right) L_{K}\left(E \cap Q_{R}, \mathcal{C} E \cap Q_{R}\right) .
$$

Proof. Let us cover the full measure of $Q_{R}$ by cubes belonging to the grid of disjoint open cubes of size $R_{0}$ given by $\left\{Q_{i}\right\} \subset R_{0}\left(\mathbb{Z}^{n}+(-1 / 2,1 / 2)^{n}\right)$. Let us consider also the covering of $Q_{R}$ by cubes in the overlapping grid of side $3 R_{0}$ given by $\left\{\bar{Q}_{i}\right\} \subset$ $R_{0}\left(\mathbb{Z}^{n}+3(-1 / 2,1 / 2)^{n}\right)$. Note that (up to sets of measure zero) each point of $Q_{R}$ belongs to exactly one cube in $\left\{Q_{i}\right\}$ and $3^{n}$ cubes in $\left\{\bar{Q}_{i}\right\}$.

Notice that for every pair of points $x, \bar{x} \in Q_{R}$ such that $|x-\bar{x}| \leqslant R_{0}$ there is some large cube $\bar{Q}_{i}$ containing at the same time both points. Indeed, $x$ will belong to some small cube $Q_{i}$ but then if $\bar{Q}_{i}$ is the large cube with the same center it will also be $y \in \bar{Q}_{i}$. Hence,

$$
\left\{(x, \bar{x}) \in Q_{R} \times Q_{R}:|x-\bar{x}| \leqslant R_{0}\right\} \subset \bigcup_{i} \bar{Q}_{i} \times \bar{Q}_{i} .
$$


This implies that

$$
\begin{aligned}
L_{K_{0}}\left(E \cap Q_{R}, \mathcal{C} E \cap Q_{R}\right) & =\iint_{\left(E \cap Q_{R}\right) \times\left(\mathcal{C} E \cap Q_{R}\right)} \chi_{\left\{|\bar{x}-x| \leqslant R_{0}\right\}} d x d \bar{x} \\
& =\iint_{\bigcup_{i}\left(E \cap \bar{Q}_{i}\right) \times\left(\mathcal{C} E \cap \bar{Q}_{i}\right)} \chi_{\left\{|\bar{x}-x| \leqslant R_{0}\right\}} d x d \bar{x} \\
& \leqslant \sum_{i} \iint_{\left(E \cap \bar{Q}_{i}\right) \times\left(\mathcal{C} E \cap \bar{Q}_{i}\right)} \chi_{\left\{|\bar{x}-x| \leqslant R_{0}\right\}} d x d \bar{x} \\
& =\sum_{i} L_{K_{0}}\left(E \cap \bar{Q}_{i}, \mathcal{C} E \cap \bar{Q}_{i}\right) .
\end{aligned}
$$

Now, using Lemma 5.1 we obtain that, for all $i$,

$$
\begin{aligned}
L_{K_{0}}\left(E \cap \bar{Q}_{i}, \mathcal{C} E \cap \bar{Q}_{i}\right) & \leqslant\left|E \cap \bar{Q}_{i}\right| \cdot\left|\mathcal{C} E \cap \bar{Q}_{i}\right| \\
& \leqslant\left(3 R_{0}\right)^{n} \min \left\{\left|E \cap \bar{Q}_{i}\right| \cdot\left|\mathcal{C} E \cap \bar{Q}_{i}\right|\right\} \\
& \leqslant C\left(n, R_{0}\right) L_{K}\left(E \cap \bar{Q}_{i}, \mathcal{C} E \cap \bar{Q}_{i}\right) .
\end{aligned}
$$

But then, using that each point of $B_{R}$ belongs to at most $3^{n}$ cubes $\bar{Q}_{i}$ we can estimate

$$
\begin{aligned}
L_{K_{0}}\left(E \cap Q_{R}, \mathcal{C} E \cap Q_{R}\right) & \leqslant \sum_{i} L_{K_{0}}\left(E \cap \bar{Q}_{i}, \mathcal{C} E \cap \bar{Q}_{i}\right) \\
& \leqslant \sum_{i} C\left(n, R_{0}\right) L_{K}\left(E \cap \bar{Q}_{i}, \mathcal{C} E \cap \bar{Q}_{i}\right) \\
& \leqslant C\left(n, R_{0}\right) 3^{n} L_{K}\left(E \cap Q_{R}, \mathcal{C} E \cap Q_{R}\right)
\end{aligned}
$$

as stated in the Lemma.

We finally give the

Proof of Proposition 1.11. Note first that all $R \geqslant 1$ we have $P_{K}\left(B_{R}\right) \geqslant c(n) R^{n-1}$ since $K \geqslant 1$ in $B_{2}$ by (1.6). On the other hand it is clear that by definition $P_{K}\left(B_{R}\right)$ is monotone in $R$.

Thus, if $k$ is the smallest integer such that $3 R_{0} k / 2 \geqslant R$, denoting $\bar{R}=3 R_{0} k / 2$, $E_{R}=E \cap B_{R}$. Denote $K_{0}(z)=\chi_{\left\{|z| \leqslant R_{0}\right\}}(z)$. Using Lemma 5.2 we obtain

$$
\begin{aligned}
P_{K_{0}}\left(E, B_{R}\right) & \leqslant L_{K_{0}}\left(E \cap B_{R}, \mathcal{C} E \cap B_{R}\right)+L_{K_{0}}\left(B_{R}, C B_{R}\right) \\
& \leqslant L_{K_{0}}\left(E_{R} \cap Q_{\bar{R}}, \mathcal{C} E_{R} \cap Q_{\bar{R}}\right)+\int_{B_{R}} \int_{\mathcal{C} B_{R}} \chi_{\left\{|\bar{x}-x| \leqslant R_{0}\right\}} d \bar{x} d x \\
& \leqslant C\left(n, R_{0}\right) L_{K}\left(E_{R} \cap Q_{\bar{R}}, \mathcal{C} E_{R} \cap Q_{\bar{R}}\right)+C\left(n, R_{0}\right) R^{n-1} \\
& \leqslant C\left(n, R_{0}\right)\left(L_{K}\left(E \cap B_{R}, \mathcal{C} E \cap B_{R}\right)+L_{K}\left(B_{R}, C B_{R}\right)+R^{n-1}\right) \\
& \leqslant C\left(n, R_{0}\right)\left(P_{K, B_{R}}(E)+P_{K}\left(B_{R}\right)+R^{n-1}\right), \\
& \leqslant C\left(n, R_{0}\right) P_{K}\left(B_{R}\right)
\end{aligned}
$$


where we have used that $E$ is a minimizer $P_{K, B_{R}}$ and hence $P_{K, B_{R}}(E) \leqslant P_{K}\left(B_{R}\right)$. Then the proposition follows.

\section{Existence AND COMPACTNESS OF Minimizers}

To prove Theorem 1.13 we need some preliminary results. First we prove existence of minimizers among "nice" sets (more precisely among sets with finite 1/2perimeter); this is done in Proposition 6.6, where a crucial ingredient in the proof is given by the uniform $B V$-bound established in Theorem 1.12 which provides the necessary compactness in $L^{1}$. Second, we establish a density result (see Proposition 6.4 which allows to approximate any set of finite $K$-perimeter, with sets that has also finite 1/2-perimeter; the proof of this density result uses a generalized coarea formula that we establish in Lemma 6.2.

We start with a simple remark which will be useful in the sequel.

Proposition 6.1 (Lower semicontinuity of $K$-perimeter). Let $\chi_{E_{k}} \rightarrow \chi_{E}$ in $L_{\text {loc }}^{1}\left(\mathbb{R}^{n}\right)$, then

$$
\liminf _{k \rightarrow \infty} P_{K, \Omega}\left(E_{k}\right) \geqslant P_{K, \Omega}(E) .
$$

Proof. The result follows, exactly as in Proposition 3.1 in [11], by Fatou Lemma. Indeed, recall that

$$
L_{K}(A, B)=\int_{\mathbb{R}^{n}} \int_{\mathbb{R}^{n}} \chi_{A}(x) \chi_{B}(\bar{x}) K(x-\bar{x}) d x d \bar{x} .
$$

If $\chi_{A_{k}} \rightarrow \chi_{A}, \chi_{B_{k}} \rightarrow \chi_{B}$ in $L_{l o c}^{1}\left(\mathbb{R}^{n}\right)$, then for each sequence there exists a subsequence $k_{j}$, such that for a.e. $(x, \bar{x})$

$$
\chi_{A_{k_{j}}} \chi_{B_{k_{j}}} \rightarrow \chi_{A} \chi_{B}
$$

Therefore, by Fatou Lemma, we have

$$
\liminf _{j \rightarrow \infty} L_{K}\left(A_{k_{j}}, B_{k_{j}}\right) \geqslant L_{K}(A, B) .
$$

In the next lemma we establish a generalized coarea formula for the $K$-perimeter. The analogue result for the fractional $s$-perimeter is contained in [43]. For the sake of completeness, we reproduce here the simple proof, which does not dependent on the choice of the kernel. For a measurable function $u$, we set:

$\mathcal{F}_{K, \Omega}(u):=\frac{1}{2} \int_{\Omega} \int_{\Omega}|u(x)-u(\bar{x})| K(x-\bar{x}) d x d \bar{x}+\int_{\Omega} \int_{\mathcal{C} \Omega}|u(x)-u(\bar{x})| K(x-\bar{x}) d x d \bar{x}$.

Lemma 6.2 (Coarea formula). Let $u: \Omega \rightarrow[0,1]$ be a measurable function. Then, we have

where $E_{t}=\{u>t\}$.

$$
\mathcal{F}_{K, \Omega}(u)=\int_{0}^{1} P_{K, \Omega}\left(E_{t}\right) d t
$$


Proof. We start by observing that the function $t \mapsto \chi_{E_{t}}(x)-\chi_{E_{t}}(\bar{x})$ takes values in $\{-1,0,1\}$ and it is different from zero in the interval having $u(x)$ and $u(\bar{x})$ as extreme points, therefore

$$
|u(x)-u(\bar{x})|=\int_{0}^{1}\left|\chi_{E_{t}}(x)-\chi_{E_{t}}(\bar{x})\right| d t .
$$

Hence, by Fubini Theorem, we deduce

$$
\begin{aligned}
\mathcal{F}_{K, \Omega}(u)= & \int_{0}^{1}\left[\frac{1}{2} \int_{\Omega} \int_{\Omega}\left|\chi_{E_{t}}(x)-\chi_{E_{t}}(\bar{x})\right| K(x-\bar{x}) d x d \bar{x}\right] d t \\
& \quad+\int_{0}^{1}\left[\int_{\Omega} \int_{\mathcal{C} \Omega}\left|\chi_{E_{t}}(x)-\chi_{E_{t}}(\bar{x})\right| K(x-\bar{x}) d x d \bar{x}\right] d t \\
= & \int_{0}^{1}\left[\int_{E_{t} \cap \Omega} \int_{\mathcal{C} E_{t} \cap \Omega} K(x-\bar{x}) d x d \bar{x}+\int_{E_{t} \cap \Omega} \int_{\mathcal{C} E_{t} \cap \mathcal{C} \Omega} K(x-\bar{x}) d x d \bar{x}\right. \\
& \left.\quad+\int_{\mathcal{C} E_{t} \cap \Omega} \int_{E_{t} \cap \mathcal{C} \Omega} K(x-\bar{x}) d x d \bar{x}\right] d t \\
= & \int_{0}^{1}\left[L_{K}\left(E_{t} \cap \Omega, \mathcal{C} E_{t} \cap \Omega\right)+L_{K}\left(E_{t} \cap \Omega, \mathcal{C} E_{t} \backslash \Omega\right)+L_{K}\left(E_{t} \backslash \Omega, \mathcal{C} E_{t} \cap \Omega\right)\right] d t \\
= & \int_{0}^{1} P_{K, \Omega}\left(E_{t}\right) d t,
\end{aligned}
$$

as desired.

In the following lemma we establish a density result for smooth functions in the space of functions with finite $\mathcal{F}_{K, \Omega}$. For the sake of completeness we reproduce here the simple proof, which follows the one in [25], Lemma 11, for the case of the all space.

Lemma 6.3. Let $\Omega$ be a bounded Lipschitz domain and $u$ be a function defined on $\mathbb{R}^{n}$ with $u \in L^{1}(\Omega)$ and $\mathcal{F}_{K, \Omega}(u)<\infty$. Then, for any fixed sufficiently small $\delta>0$, there exists a family $\left(u_{\varepsilon}\right)$ of smooth functions such that:

i) $\left\|u-u_{\varepsilon}\right\|_{L^{1}\left(\Omega^{\delta}\right)} \rightarrow 0$ as $\varepsilon \rightarrow 0$,

ii) $\mathcal{F}_{K, \Omega^{\delta}}\left(u-u_{\varepsilon}\right) \rightarrow 0$ as $\varepsilon \rightarrow 0$.

Proof. For any $0<\varepsilon<\delta$, we consider the convolution kernel

$$
\eta_{\varepsilon}(x):=\varepsilon^{-n} \eta\left(\frac{x}{\varepsilon}\right)
$$

where $\eta \in C_{0}^{\infty}\left(B_{1}\right), \eta \geqslant 0, \int_{\mathbb{R}^{n}} \eta=1$, and we set

$$
u_{\varepsilon}(x):=\left(u * \eta_{\varepsilon}\right)(x) .
$$

Since $u \in L^{1}(\Omega)$ we immediately have $\left\|u-u_{\varepsilon}\right\|_{L^{1}\left(\Omega^{\delta}\right)} \rightarrow 0$ as $\varepsilon \rightarrow 0$. It remains to prove ii). 
Using the definition of $u_{\varepsilon}$ and the triangle inequality, we have that

$$
\begin{aligned}
& 2 \mathcal{F}_{K, \Omega^{\delta}}\left(u_{\varepsilon}-u\right)=\iint_{\mathbb{R}^{2 n} \backslash\left(\mathcal{C} \Omega^{\delta} \times \mathcal{C} \Omega^{\delta}\right)}\left|u_{\varepsilon}(x)-u(x)+u(\bar{x})-u_{\varepsilon}(\bar{x})\right| K(x-\bar{x}) d x d \bar{x} \\
& \quad=\iint_{\mathbb{R}^{2 n} \backslash\left(\mathcal{C} \Omega^{\delta} \times \mathcal{C} \Omega^{\delta}\right)} K(x-\bar{x})\left|\int_{B_{1}}(u(x-\varepsilon z)-u(\bar{x}-\varepsilon z)-u(x)+u(\bar{x})) \eta(z) d z\right| d x d y \\
& \quad \leqslant \int_{B_{1}} \iint_{\mathbb{R}^{2 n} \backslash\left(\mathcal{C} \Omega^{\delta} \times \mathcal{C} \Omega^{\delta}\right)} K(x-\bar{x})|u(x-\varepsilon z)-u(\bar{x}-\varepsilon z)-u(x)+u(\bar{x})| \eta(z) d x d y d z .
\end{aligned}
$$

Now, by the continuity of translations in $L^{1}\left(\mathbb{R}^{2 n} \backslash\left(C \Omega^{\delta} \times \mathcal{C} \Omega^{\delta}\right)\right)$ applied to the function

$$
v(x, \bar{x})=(u(x)-u(\bar{x})) K(x-\bar{x}),
$$

(which is in $L^{1}\left(\mathbb{R}^{2 n} \backslash\left(C \Omega^{\delta} \times \mathcal{C} \Omega^{\delta}\right)\right.$ ), since $\left.\mathcal{F}_{K, \Omega}(u)<\infty\right)$, we deduce that for every fixed $z \in B_{1}$,

$\iint_{\mathbb{R}^{2 n} \backslash\left(\mathcal{C} \Omega^{\delta} \times \mathcal{C} \Omega^{\delta}\right)} K(x-\bar{x})|u(x-\varepsilon z)-u(\bar{x}-\varepsilon z)-u(x)+u(\bar{x})| d x d y \rightarrow 0, \quad$ as $\quad \varepsilon \rightarrow 0$.

Moreover, for a.e. $z \in B_{1}$, we have

$$
\begin{aligned}
& \eta(z) \iint_{\mathbb{R}^{2 n} \backslash\left(\mathcal{C} \Omega^{\delta} \times \mathcal{C} \Omega^{\delta}\right)} K(x-\bar{x})|u(x-\varepsilon z)-u(\bar{x}-\varepsilon z)-u(x)+u(\bar{x})| d x d y \\
& \leqslant 2 \max \eta \iint_{\mathbb{R}^{2 n} \backslash\left(\mathcal{C} \Omega^{\delta} \times \mathcal{C} \Omega^{\delta}\right)} K(x-\bar{x})|u(x)-u(\bar{x})| d x d y<\infty .
\end{aligned}
$$

Hence, the conclusion follows by the dominated convergence Theorem.

The following density result will be useful in the proof of existence of minimizers. The proof follows the one for the classical approximation result for sets of finite perimeter by smooth sets, and uses the generalized coarea formula of Lemma 6.2.

Proposition 6.4 (Density of sets with finite 1/2-perimeter). Let $\Omega$ be $a$ bounded Lipschitz domain. Let $F$ be a set with finite $K$-perimeter in $\Omega$. Then, there exists a sequence $\left(F_{j}\right)$ of open sets satisfying the following properties:

(1) $P_{1 / 2, \Omega}\left(F_{j}\right)<\infty$

(2) $F_{j} \backslash \Omega=F \backslash \Omega$,

(3) $\lim _{j \rightarrow \infty}\left|F_{j} \triangle F\right|=0$

(4) $\lim _{j \rightarrow \infty} P_{K, \Omega}\left(F_{j}\right)=P_{K, \Omega}(F)$.

To prove Proposition 6.4, we need the following preliminary result.

Let $\Omega$ be a Lipschitz domain and let $d(x, \partial \Omega)$ denote the distance of the point $x$ from the boundary $\partial \Omega$. We define

$$
\Omega^{t}:=\{x \in \Omega: d(x, \partial \Omega)>t\} \quad \text { and }
$$

Note that for a sufficiently small $\delta_{0}>0$, where $t \in\left(0, \delta_{0}\right)$ all the domains $\Omega^{t}$ are Lipschitz with uniform constants depending only on $\Omega$. We will need the following lemma. 
Lemma 6.5. Let $\Omega \subset \mathbb{R}^{n}$ be a Lipschitz domain and suppose that $K \geqslant 0$ satisfies assumption 1.5). There exists $\delta_{0}>0$ depending only on $\Omega$ such that for any $t \in\left(0, \delta_{0}\right)$ we have

$$
L_{K}\left(\Omega \backslash \Omega^{t}, \Omega^{t}\right) \leqslant C \int_{\mathbb{R}^{n}} \min \{t,|z|\} K(z) d z,
$$

and

$$
L_{K}\left(\Omega \backslash \Omega^{t}, \mathcal{C} \Omega\right) \leqslant C \int_{\mathbb{R}^{n}} \min \{t,|z|\} K(z) d z,
$$

where the constants $C$ and $\delta$ depend only on $\Omega$.

Proof. Performing the change of variables $z=\bar{x}-x$ and using Fubini Theorem, we have

$$
\begin{aligned}
L_{K}\left(\Omega \backslash \Omega^{t}, \Omega^{t}\right) & =\int_{\Omega^{t}} d x \int_{\Omega \backslash \Omega^{t}} d \bar{x} K(\bar{x}-x)=\int_{\mathbb{R}^{n}} d z K(z) \int_{\Omega^{t} \cap\left(\left(\Omega \backslash \Omega^{t}\right)-z\right)} d x \\
& \leqslant C \int_{\mathbb{R}^{n}} \min \{t,|z|\} K(z) d z,
\end{aligned}
$$

since for a Lipschitz set $\Omega$, we have

$$
\left|\Omega^{t} \cap\left(\left(\Omega \backslash \Omega^{t}\right)-z\right)\right| \leqslant \min \left\{\left|\Omega \backslash \Omega^{t}\right|,\left|\Omega^{t} \backslash\left(\Omega^{t}-z\right)\right|\right\} \leqslant \min \{t,|z|\} .
$$

The proof of 6.3 follows likewise.

Proof of Proposition 6.4. As it will become clear in the proof, actually we prove more than property (1): we will show that for any $j$, on the one hand $\partial F_{j}$ is smooth in $\Omega^{\frac{1}{j}}$ (and up to the boundary of $\Omega^{\frac{1}{j}}$ ), and on the other hand $F_{j} \cap \Omega \backslash \Omega^{\frac{1}{j}}$. Recall that $\Omega^{\frac{1}{j}}$ was defined in (6.1). Since $\Omega$ is Lipschitz, these two properties imply that $F_{j}$ satisfies (1), for $j$ large enough.

For a fixed sufficiently small $\delta$, we consider $\Omega^{\delta}$-as in 6.1). Let $\varepsilon_{k} \in(0, \delta)$ be a sequence such that $\varepsilon_{k} \downarrow 0$, and let $u_{k}$ be the mollified functions

$$
u_{k}:=\chi_{F} * \eta_{\varepsilon_{k}} .
$$

By Lemma 6.3 we know that

$$
\left\|u_{k}-\chi_{F}\right\|_{L^{1}\left(\Omega^{\delta}\right)} \rightarrow 0, \quad \text { as } k \rightarrow \infty,
$$

and

$$
\lim _{k \rightarrow \infty} \mathcal{F}_{K, \Omega^{\delta}}\left(u_{k}\right)=\mathcal{F}_{K, \Omega^{\delta}}\left(\chi_{F}\right)=P_{K, \Omega^{\delta}}(F) .
$$

We define now the sets

$$
F_{t}^{k}:=\left\{u_{k}>t\right\} .
$$

By the coarea formula of Lemma 6.2, we have that

$$
\begin{aligned}
P_{K, \Omega^{\delta}}(F) & =\lim _{k \rightarrow \infty} \mathcal{F}_{K, \Omega^{\delta}}\left(u_{k}\right) \\
& \geqslant \int_{0}^{1} \liminf _{k \rightarrow \infty} P_{K, \Omega^{\delta}}\left(F_{t}^{k}\right) d t .
\end{aligned}
$$


Sard's Theorem implies that for $\mathcal{L}^{1}$-a.e. $t \in(0,1)$, all the sets $F_{t}^{k}$ have smooth boundary, therefore we can choose $t$ with this property and such that

$$
L:=\liminf _{k \rightarrow \infty} P_{K, \Omega^{\delta}}\left(F_{t}^{k}\right) \leqslant P_{K, \Omega^{\delta}}(F) .
$$

Let now $\left(F_{h}\right)=\left(F_{t}^{k(h)}\right)$ be a subsequence with finite $K$-Perimeter in $\Omega^{\delta}$ converging to $L$. By Chebyshev inequality and (6.4) we deduce that

$$
\left|\left(F_{h} \triangle F\right) \cap \Omega^{\delta}\right| \rightarrow 0, \quad \text { as } h \rightarrow \infty .
$$

Moreover by the lower semicontinuity of the $K$-Perimeter, we deduce that

$$
\lim _{h \rightarrow \infty} P_{K, \Omega^{\delta}}\left(F_{h}\right)=P_{K, \Omega^{\delta}}(F) .
$$

We define now the sequence of sets

$$
F_{h}^{\delta}:=\left(F_{h} \cap \Omega^{\delta}\right) \cup A^{\delta} \cup(F \backslash \Omega) .
$$

We start by observing that, by definition, $F_{h}^{\delta}$ satisfies

$$
F_{h}^{\delta} \backslash \Omega=F \backslash \Omega \text {, and } F_{h}^{\delta} \text { is smooth in } \overline{\Omega^{\delta}} .
$$

Moreover, using Lemma 6.3 and that $\left|A^{\delta}\right|=C \delta$, we see that

$$
\lim _{h \rightarrow \infty}\left|F_{h}^{\delta} \triangle F\right|=C \delta .
$$

Here and in the sequel $C$ denotes possibly different positive constant (uniform in $h$ and $\delta$ ). We estimate now how much the $K$-perimeters of $F$ and $F_{h}^{\delta}$ differs. By the triangle inequality, we have that

$$
\begin{aligned}
\left|P_{K, \Omega}\left(F_{h}^{\delta}\right)-P_{K, \Omega}(F)\right| \leqslant & \left|P_{K, \Omega}\left(F_{h}^{\delta}\right)-P_{K, \Omega^{\delta}}\left(F_{h}^{\delta}\right)\right|+\left|P_{K, \Omega^{\delta}}\left(F_{h}^{\delta}\right)-P_{K, \Omega^{\delta}}\left(F_{h}\right)\right|+ \\
& \quad+\left|P_{K, \Omega^{\delta}}\left(F_{h}\right)-P_{K, \Omega^{\delta}}(F)\right|+\left|P_{K, \Omega^{\delta}}(F)-P_{K, \Omega}(F)\right| \\
= & I_{1}+I_{2}+I_{3}+I_{4} .
\end{aligned}
$$

We readiy show that, for $i=1,2,4$,

$$
I_{i} \leqslant L_{K}\left(\Omega \backslash \Omega^{\delta}, \Omega^{\delta}\right)+L_{K}\left(\Omega \backslash \Omega^{\delta}, \mathcal{C} \Omega^{\delta}\right) .
$$

Using Lemma 6.5 we deduce that

$$
I_{i} \leqslant C \int_{\mathbb{R}^{n}} K(z) \min \{\delta,|z|\} d z
$$

where $C$ depends only on $\Omega$. Finally, by point (4) in Proposition 6.4, we have that for any fixed $\delta$,

$$
I_{3} \rightarrow 0 \quad \text { as } h \rightarrow \infty
$$


Let now $j$ be given. We choose $\delta=\delta(j)$ such that $I_{i} \leqslant 1 /(4 j)$, for $i=1,2,4$. Moreover, by 6.12 , we can choose $h=h(j)$ such that $I_{3} \leqslant 1 /(4 j)$. Finally we set $F_{j}:=F_{h(j)}^{\delta(j)}$. With this choices, plugging 6.11, 6.12 in 6.10 we deduce that

$$
\left|P_{K, \Omega}\left(F_{j}\right)-P_{K, \Omega}(F)\right| \leqslant \frac{1}{j} .
$$

In addition, by 6.8 and 6.9 , we have that $F^{j}$ has smooth boundary in $\Omega^{\frac{1}{j}}$ and is such that

$$
F_{j} \backslash \Omega=F \backslash \Omega, \quad\left|F_{j} \triangle F\right| \leqslant \frac{1}{j} .
$$

To conclude the proof, it remains therefore to show (1). This is an easy consequence of the fact that $F_{j}$ has smooth boundary in $\Omega^{\frac{1}{j}}$. Indeed, given any set $\tilde{F}$ with smooth boundary in $\Omega^{\frac{1}{j}}$, and using again Lemma 6.5. we have

$$
\begin{aligned}
P_{1 / 2, \Omega}(\tilde{F}) & =P_{1 / 2, \Omega^{\frac{1}{j}}}(\tilde{F})+C \int_{\mathbb{R}^{n}} K(z) \min \left\{\frac{1}{j},|z|\right\} d z \\
& =\int_{\tilde{F} \cap \Omega^{\frac{1}{j}}} \int_{\mathcal{C} \tilde{F} \cap \Omega^{\frac{1}{j}}} \frac{1}{|x-\bar{x}|^{n+s}} d x d \bar{x}+2 \int_{\Omega^{\frac{1}{j}}} \int_{\mathcal{C} \Omega^{\frac{1}{j}}} \frac{1}{|x-\bar{x}|^{n+s}} d x d \bar{x}+C<\infty,
\end{aligned}
$$

as desired.

Proposition 6.6 (Existence of minimizers among "nice" sets). Let $\Omega$ be a bounded Lipschitz domain, and $E_{0} \subset \mathcal{C} \Omega$ a given set. Then, there exists a set $E$, with $E \cap \mathcal{C} \Omega=E_{0}$ that is a minimizer for $P_{K, \Omega}$ among all sets $F$ with $P_{1 / 2, \Omega}(F) \leqslant C$.

Proof. Let $\varepsilon>0$. We introduce the following regularized kernel:

$$
K_{\varepsilon}(z):=K(z)+\frac{\varepsilon}{|z|^{n+\frac{1}{2}}} .
$$

For any $\varepsilon$ fixed, the associated perimeter $P_{K_{\varepsilon}, \Omega}$ admits a minimizer $E_{\varepsilon}$ with $E_{\varepsilon} \cap$ $\mathcal{C} \Omega=E_{0}$. This follows as in the proof of Theorem 3.2 in [11] by $L^{1}$-compactness of $H^{\frac{1}{4}}$ and the lower semicontinuity of $P_{K_{\varepsilon}, \Omega}$ (that follows by Proposition 6.1 applied to $P_{K_{\varepsilon}, \Omega}$ in place of $\left.P_{K, \Omega}\right)$. Indeed given $F_{\varepsilon, k}$ a sequence of sets such that

$$
P_{K_{\varepsilon}, \Omega}\left(F_{\varepsilon, k}\right) \underset{k \rightarrow \infty}{\longrightarrow} \inf _{F \cap \mathcal{C} \Omega=E_{0}} P_{K_{\varepsilon}, \Omega}(F),
$$

then the $H^{\frac{1}{4}}$-norm of the characteristic functions of $F_{\varepsilon, k} \cap \Omega$ are bounded (by a constant depending on $\varepsilon$ ), thus, by compactness, there exists a subsequence which converges to a set $E_{\varepsilon} \cap \Omega$ in $L^{1}\left(\mathbb{R}^{n}\right)$, which is a minimizer of $P_{K_{\varepsilon}, \Omega}$ by lower semicontinuity.

Now we observe that the new kernel $K_{\varepsilon}$ satisfies all assumptions $(1.3)-(1.6)$ and (1.7), therefore, by Theorem 1.12 and a standard covering argument, we have a uniform $B V$-bound (uniform in $\varepsilon$ !) for the characteristic functions of the minimizers $E_{\varepsilon}$ in any subdomains $\Omega^{\prime}$, with $\overline{\Omega^{\prime}} \subset \Omega$. We set, as above, $\Omega^{\delta}=\{x \in \Omega: d(x, \partial \Omega)>$ $\delta\}$. 
Using that $B V$ is compact in $L^{1}$ and the standard diagonal argument, we can extract a subsequence $\varepsilon_{j}$ such that

$$
\chi_{E_{\varepsilon_{j}}} \rightarrow \chi_{E} \text { in } L^{1}\left(\Omega^{\delta}\right) \text { for all } \delta>0 .
$$

It remains to prove that $E$ is a minimizer for $P_{K, \Omega}$. On one hand, by definition of $K_{\varepsilon}$ and by the lower semicontinuity of $P_{K, \Omega}$, we have

$$
\liminf _{\varepsilon \rightarrow 0} P_{K_{\varepsilon}, \Omega^{\delta}}\left(E_{\varepsilon}\right) \geqslant \liminf _{\varepsilon \rightarrow 0} P_{K, \Omega^{\delta}}\left(E_{\varepsilon}\right) \geqslant P_{K, \Omega^{\delta}}(E) .
$$

On the other hand, by minimality of $E_{\varepsilon}$, we have that

$$
P_{K_{\varepsilon}, \Omega}\left(E_{\varepsilon}\right) \leqslant P_{K_{\varepsilon}, \Omega}(F),
$$

for any measurable set $F$ with $F \cap \mathcal{C} \Omega=E_{0}$.

Hence, we deduce that

$$
\begin{aligned}
P_{K, \Omega^{\delta}}(E) & \leqslant \liminf _{\varepsilon \rightarrow 0} P_{K_{\varepsilon}, \Omega^{\delta}}\left(E_{\varepsilon}\right) \\
& \leqslant P_{K_{\varepsilon}, \Omega}(F) \\
& =P_{K, \Omega}(F)+\varepsilon P_{1 / 2, \Omega}\left(F_{\delta}\right)
\end{aligned}
$$

When a $P_{1 / 2, \Omega}(F)<\infty$, the conclusion then follows by sending first $\varepsilon$ to zero and then $\delta$ to zero.

We can now give the proof of our existence result.

Proof of Theorem 1.13. The theorem follows combining Propositions 6.6 and 6.4.

Lemma 6.7 (Compactness). Let $\Omega$ be a Lipschitz domain in $\mathbb{R}^{n}$. Assume that $K$ satisfies (1.3), (1.4), (1.5) and (1.6). Let $\left\{E_{n}\right\}$ be a minimizing sequence for $P_{K, \Omega}$ and

$$
\chi_{E_{k}} \rightarrow \chi_{E} \quad \text { in } L_{l o c}^{1}\left(\mathbb{R}^{n}\right) .
$$

Then, $E$ is a minimizer for $P_{K, \Omega}$ and

$$
\lim _{k \rightarrow \infty} P_{K, \Omega}\left(E_{k}\right)=P_{K, \Omega}(E) .
$$

Proof. We follow the proof of Theorem 3.3 in [11].

Assume that $F=E$ outside $\Omega$. We set

$$
F_{k}:=(F \cap \Omega) \cup\left(E_{k} \backslash \Omega\right),
$$

then, by minimality of $E_{k}$, we have

$$
P_{K, \Omega}\left(F_{k}\right) \geqslant P_{K, \Omega}\left(E_{k}\right) .
$$

Moreover, by definition of $F_{k}$

$$
\left|P_{K, \Omega}(F)-P_{K, \Omega}\left(F_{k}\right)\right| \leqslant L_{K}\left(\Omega,\left(E_{k} \triangle E\right) \backslash \Omega\right) .
$$

We denote:

$$
b_{k}:=L_{K}\left(\Omega,\left(E_{k} \triangle E\right) \backslash \Omega\right),
$$


and we get

$$
P_{K, \Omega}(F)+b_{k} \geqslant P_{K, \Omega}\left(E_{k}\right)
$$

To conclude we just need to prove that $b_{k} \rightarrow 0$ as $k \rightarrow \infty$, indeed, by lower semicontinuity, we would deduce that

$$
P_{K, \Omega}(F) \geqslant \limsup _{k \rightarrow \infty} P_{K, \Omega}\left(E_{k}\right) \geqslant \liminf _{k \rightarrow \infty} P_{K, \Omega}\left(E_{k}\right) \geqslant P_{K, \Omega}(E) .
$$

Finally we observe that, by Remark 1.4 , we have that the function

$$
\phi(\bar{x}):=\int_{\Omega} K(x-\bar{x}) d x
$$

belongs to $L^{1}(\mathcal{C} \Omega)$. Then, using that $\chi_{E_{k}} \rightarrow \chi_{E}$ in $L_{l o c}^{1}$ as $k \rightarrow \infty$, the dominated convergence theorem implies

$$
b_{k}=\int_{\left(E_{k} \triangle E\right) \backslash \Omega} \int_{\Omega} K(x-\bar{x}) d x \rightarrow 0, \quad \text { as } k \rightarrow \infty,
$$

which concludes the proof.

\section{APPENDIX: INTEGRAL FORMUlas FOR SETS OF FINITE PERIMETER}

We sketch here the

Proof of Proposition 4.4. We follow Section 5.10.2 in the book of Evans and Gariepy 23.

Step 1. We show that the map $L^{\perp} \rightarrow \mathbb{R}$

$$
y \mapsto I_{E, \Omega}(L, y)
$$

is $H^{n-m}$ measurable. This follows exactly as in the proof of [23, Lemma 1 §5.10.2] using that the supremum in the definition of $I_{E, \Omega}(L, y)$ in 4.5$)$ is actually the supremum $\phi$ belonging to a countable dense subset of $C_{c}^{1}\left((y+L) \cap \Omega ; L \cap B_{1}\right)$.

Step 2. We prove that

$$
\int_{L^{\perp}} I_{E, \Omega}(L, y) d H^{n-m}(y) \leqslant\left|\nabla_{L} u\right|(\Omega),
$$

where we recall that $u=\chi_{E}$ is a function in $\operatorname{BV}(\Omega)$ and

$$
\left|\nabla_{L} u\right|(\Omega):=\sup \left\{\int_{\Omega} u(x) \operatorname{div} \phi(x) d x: \phi \in C_{c}^{1}\left(\Omega ; L \cap B_{1}\right)\right\}
$$

is the total variation of the projection of the (vector valued) measure $\nabla u$ onto $L$.

Let $\Omega^{\prime} \subset \subset \Omega$. Define given $r>0$ define $u_{r}=u * \eta_{r}$ where $\eta_{r}=r^{-n}(\dot{\bar{r}}) \geqslant 0$ is a standard smooth mollifier. Note that for $r$ small enough (depending on $\Omega^{\prime}$ ) we have

$$
\int_{\Omega^{\prime}}\left|\nabla_{L} u_{r}\right| d x \leqslant\left|\nabla_{L} u\right|(\Omega)
$$

where $\nabla_{L}$ denotes the projection of the gradient onto $L$. 
Similarly as in the proof of [23, Theorem 2 \$5.10.2], for $H^{n-m}$ a.e. $y \in L^{\perp}$, we have $u_{r} \rightarrow u$ in $L^{1}$ when the two functions are restricted to the cap $\Omega \cap(y+L)$. Hence, for $H^{n-m}$ a.e. $y$ we have

$$
I_{E, \Omega^{\prime}}(L, y) \leqslant \liminf _{r \rightarrow 0} \int_{\Omega^{\prime} \cap(y+L)}\left|\nabla_{L} u_{r}\right| d z .
$$

Thus, Fatou's Lemma implies

$$
\begin{aligned}
\int_{L^{\perp}} I_{E, \Omega^{\prime}}(L, y) d y & \leqslant \liminf _{r \rightarrow 0} \int_{L^{\perp}} d y \int_{\Omega^{\prime} \cap(y+L)} d z\left|\nabla_{L} u_{r}\right|(z) \\
& =\int_{\Omega^{\prime}}\left|\nabla_{L} u_{r}\right| d x \leqslant\left|\nabla_{L} u\right|(\Omega) .
\end{aligned}
$$

Then, 6.15 follows by monotone convergence letting $\Omega^{\prime} \uparrow \Omega$.

Step 3. We prove that

$$
\left|\nabla_{L} u\right|(\Omega) \leqslant \int_{L^{\perp}} I_{E, \Omega}(L, y) d H^{n-m}(y) .
$$

Indeed, using the definition of $I_{E, \Omega}(L, y)$ we find that for every given $\phi \in C_{c}^{1}(\Omega ; L \cap$ $B_{1}$ ) we have

$$
\int_{\Omega} u(x) \operatorname{div} \phi(x) d x \leqslant \int_{L^{\perp}} I_{E, \Omega}(L, y) d H^{n-m}(y) .
$$

Taking the supremum in $\phi$ we obtain 6.16.

Step 4. We show that

$$
\left|\nabla_{L} u\right|(\Omega)=\int_{\partial^{*} E \cap \Omega} \sqrt{\sum_{i=1}^{m}\left(\boldsymbol{v}_{i} \cdot \nu_{E}(z)\right)^{2}} d H^{n-1}(z) .
$$

To prove 6.17) we use the divergence theorem for the set of finite perimeter $E$ and with a vector field $\phi \in C_{c}^{1}\left(\Omega ; L \cap B_{1}\right)$. We obtain

$$
\begin{aligned}
\int_{\Omega} u(x) \operatorname{div} \phi(x) d x & =\int_{\partial^{*} E \cap \Omega} \phi(z) \cdot \nu_{E}(z) d H^{n-1}(z) \\
& \leqslant \int_{\partial^{*} E \cap \Omega} \sqrt{\sum_{i=1}^{m}\left(\boldsymbol{v}_{i} \cdot \nu_{E}(z)\right)^{2}} d H^{n-1}(z) .
\end{aligned}
$$

From this, taking supremums in the left hand side, it easily follows that (6.17) is satisfied with the equality sign replaced by $\leqslant$. To prove the equality we may use the structure theorem for sets of finite perimeter to build a sequence $\phi_{k}$ that attain, in the limit, the equality case in (6.18). More precisely, this follows in a rather straightforward way from the fact that $\partial^{*} E$ is $H^{n-1}$ rectifiable - see statements (i) and (ii) of Theorem 2 in Section 5.7 .3 of [23]. 
Step 4. In the case of $m=1$ the formulas for $I_{E, \Omega}(L, y)$ and $I_{E, \Omega}(L, y)_{ \pm}$follow by inspection using the fact that a set of finite perimeter in dimension one is (up to negligible sets) a finite union of disjoint closed intervals.

\section{ACKNOWLEDGEMENTS}

It is a pleasure to thank Xavier Cabré, Francesco Maggi, and Albert Mas for interesting conversations on the content of this paper.

This work was supported by ERC grant 277749 "E.P.S.I.L.O.N. Elliptic PDE's and Symmetry of Interfaces and Layers for Odd Nonlinearities" and PRIN grant 201274FYK7 "Critical Point Theory and Perturbative Methods for Nonlinear Differential Equations".

\section{REFERENCES}

[1] G. Alberti, G. Bouchitté, P. Seppecher, Phase transition with the line-tension effect, Arch. Rational Mech. Anal. 144 (1998), no. 1, 1-46.

[2] L. Ambrosio, G. De Philippis, and L. Martinazzi, Gamma-convergence of nonlocal perimeter functionals, Manuscripta Math. 134 (2011), no. 3-4, 377-403.

[3] G. Barles and C. Georgelin, A simple proof of convergence for an approximation scheme for computing motions by mean curvature, SIAM J. Numer. Anal. 32 (1995), no. 2, 484-500.

[4] B. Barrios,A. Figalli, and E. Valdinoci, Bootstrap regularity for integro-differential operators and its application to nonlocal minimal surfaces, Ann. Sc. Norm. Super. Pisa Cl. Sci. (5) 13 (2014), no. 3, 609-639.

[5] H. Brezis, How to recognize constant functions. A connection with Sobolev spaces, Uspekhi Mat. Nauk 57 (2002), no. 4(346), 59-74.

[6] X. Cabré and E. Cinti, Sharp energy estimates for nonlinear fractional diffusion equations, Calc. Var. Partial Differential Equations 49 (2014), no. 1-2, 233-269.

[7] X. Cabré and E. Cinti, Energy estimates and 1-D symmetry for nonlinear equations involving the half-Laplacian, Discrete Contin. Dyn. Syst. 28 (2010), no. 3, 1179-1206.

[8] X. Cabré and Y. Sire, Nonlinear equations for fractional Laplacians II: Existence, uniqueness, and qualitative properties of solutions, Trans. Amer. Math. Soc. 367 (2015), no. 2, 911-941.

[9] X. Cabré and Y. Sire, Nonlinear equations for fractional Laplacians, I: Regularity, maximum principles, and Hamiltonian estimates, Ann. Inst. H. Poincaré Anal. Non Linéaire 31 (2014), no. $1,23-53$.

[10] X. Cabré and J. Sola-Morales, Layer solutions in a half-space for boundary reactions, Comm. Pure Appl. Math. 58 (2005), no. 12, 1678-1732.

[11] L. Caffarelli, J.-M. Roquejoffre, and O. Savin, Nonlocal minimal surfaces, Comm. Pure Appl. Math. 63 (2010), 1111-1144.

[12] L. Caffarelli and L. Silvestre, Regularity theory for fully nonlinear integro-differential equations, Comm. Pure Appl. Math. 62 (2009), no. 5, 597-638.

[13] L. Caffarelli and P. Souganidis, Convergence of Nonlocal Threshold Dynamics Approximations to Front Propagation, Arch. Rational Mech. Anal. 195 (2010), 1-23.

[14] L. Caffarelli and E. Valdinoci, Uniform estimates and limiting arguments for nonlocal minimal surfaces, Calc. Var. Partial Differential Equations 41 (2011), no. 1-2, 203-240.

[15] L. Caffarelli and E. Valdinoci, Regularity properties of nonlocal minimal surfaces via limiting arguments, Adv. Math. 248 (2013), 843-871.

[16] A. Chambolle, M. Morini, and M Ponsiglione, Nonlocal curvature flows. Arch. Ration. Mech. Anal. 218 (2015), no. 3, 1263-1329. 
[17] A. Chambolle and M. Novaga, Convergence of an algorithm for the anisotropic and crystalline mean curvature flow, SIAM J. Math. Anal. 37 (2006), no. 6, 1978-1987.

[18] T. H. Colding, W. P. Minicozzi II Estimates for parametric elliptic integrands, Int. Math. Res. Not. 2002, no. 6, 291-297.

[19] J. Dávila, On an open question about functions of bounded variation, Calc. Var. Partial Differential Equations 15 (2002), no. 4, 519-527.

[20] M. do Carmo and C. K. Peng. Stable minimal surfaces in $R^{3}$ are planes, Bulletin of the AMS (1979), 903-906.

[21] E. Di Nezza, G. Palatucci, and E. Valdinoci, Hitchhiker's guide to the fractional Sobolev spaces, (English summary) Bull. Sci. Math. 136 (2012), no. 5, 521-573.

[22] L.C. Evans, Convergence of an algorithm for mean curvature motion, Indiana Univ. Math. J. 42 (1993), no. 2, 533-557.

[23] L. C. Evans, R. F. Gariepy, Measure Theory and Fine Properties of Functions, Studies in Advanced Mathematics, 5. CRC Press (1991).

[24] A. Figalli, N. Fusco, F. Maggi, V. Millot, and M. Morini, Isoperimetry and stability properties of balls with respect to nonlocal energies. Comm. Math. Phys. 336 (2015), no. 1, 441-507.

[25] A. Fiscella, R. Servadei, and E. Valdinoci, Density properties for fractional Sobolev spaces, Ann. Acad. Sci. Fenn. Math. 40 (2015), no. 1, 235-253.

[26] D. Fischer-Colbrie and R. Schoen. The structure of complete stable minimal surfaces in 3manifolds of non-negative scalar curvature, Comm. on Pure and Appl. Math., 33 (1980), 199-211.

[27] E. Giusti, Minimal surfaces and functions of bounded variation, Monographs in Mathematics, 80. Birkhauser Verlag, Basel (1984).

[28] C. Imbert, Level set approach for fractional mean curvature flows, Interfaces Free Bound. 11 (2009), no. 1, 153-176.

[29] H. Ishii, G.E. Pires, and P.E. Souganidis, Threshold dynamics type approximation schemes for propagating fronts, J. Math. Soc. Japan 51 (1999), no. 2, 267-308.

[30] M. Ludwig, Anisotropic Fractional Perimeters, J. Differential Geom., 96 (2014), no. 1, 77-93.

[31] F. Maggi, Sets of finite perimeter and geometric variational problems. An introduction to geometric measure theory, Cambridge Studies in Advanced Mathematics, 135. Cambridge University Press, Cambridge, (2012).

[32] H.W. Meeks III, Proofs of some classical theorems in minimal surface theory, Indiana Univ. Math. J. 54 (2005), no. 4, 1031-1045.

[33] B. Merriman, J.K. Bence, and S. J. Oscher. Diffusion generated motion by mean curvature, in Computational Crystal Growers Workshop, J.E. Taylo, ed., Del Lectures Math., AMS, Providence, RI (1992), 73-83.

[34] L. Modica and S. Mortola, Un esempio di G-convergenza, (Italian) Boll. Un. Mat. Ital. B (5) 14 (1977), no. 1, 285-299.

[35] A. V. Pogorelov. On the stability of minimal surfaces, Soviet Math. Dokl., 24 (1981), 274-276.

[36] M. Sáez and E. Valdinoci, On the evolution by fractional mean curvature, preprint. (Available at http://arxiv.org/pdf/1511.06944.pdf).

[37] O. Savin and E. Valdinoci, Regularity of nonlocal minimal cones in dimension 2, Calc. Var. Partial Differential Equations, 48 (2013), no. 1-2, 33-39.

[38] O. Savin and E. Valdinoci, Some monotonicity results for minimizers in the calculus of variations, J. Funct. Anal. 264 (2013), no. 10, 2469-2496.

[39] O. Savin and E. Valdinoci, G-convergence for nonlocal phase transitions, Ann. Inst. H. Poincaré Anal. Non Lineaire 29 (2012), no. 4, 479-500.

[40] O. Scherzer, M. Grasmair, H. Grossauer, M. Haltmeier and F. Lenzen, Variational methods in imaging, Applied Mathematical Sciences, 167. Springer, New York (2009). 
[41] L. Simon, Schauder estimates by scaling, Calc. Var. Partial Differential Equations 5 (1997), no. 5, 391-407.

[42] Y. Sire and E. Valdinoci,Fractional Laplacian phase transitions and boundary reactions: a geometric inequality and a symmetry result, J. Funct. Anal. 256 (2009), no. 6, 1842-1864.

[43] A. Visintin, Generalized coarea formula and fractal sets, Japan J. Indust. Appl. Math., 8 (1991), 175-201.

[44] B. White, Lectures on Minimal Surface Theory, arXiv:1308.3325.

[45] J. A. Wieacker, Translative Poincaré formulae for Hausdorff rectifiable sets, Geom. Dedicata 16 (1984), no. 2, 231-248.

E.C, J.S, E.V, Wierstrass Institute for Applied Analysis and Stochastics, MohrenSTR. 39, 10117 BERLIN (GERMANY)

E-mail address: cinti@wias-berlin.de, serra@wias-berlin.de, valdinoc@wias-berlin.de 